$\begin{array}{llll}\text { Al-Rafidain Engineering } \quad \text { Vol.13 No.4 } & 2005\end{array}$

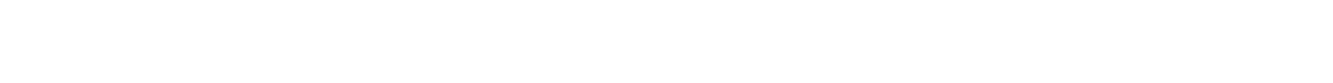

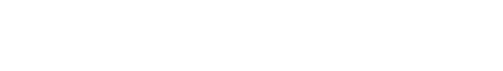

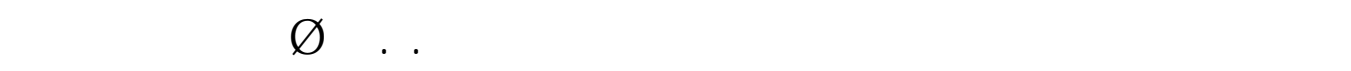

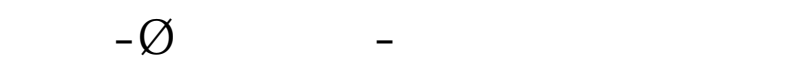

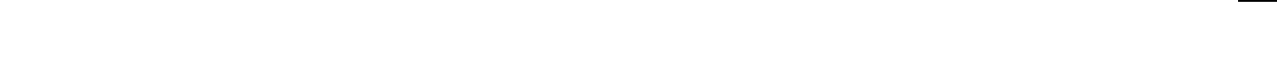

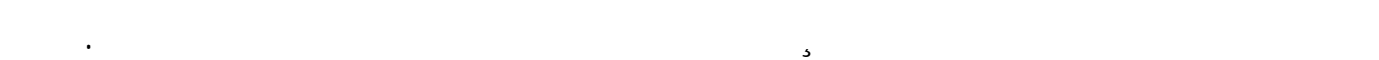

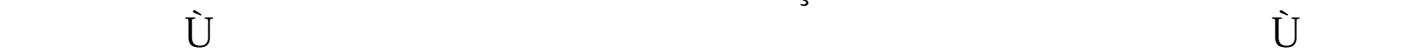

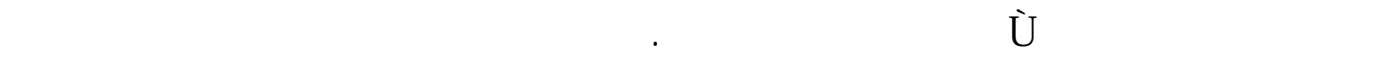

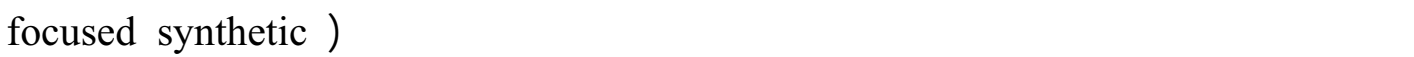

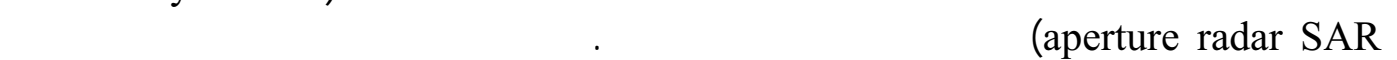
řŷũŭ ü УFś ĐŌ

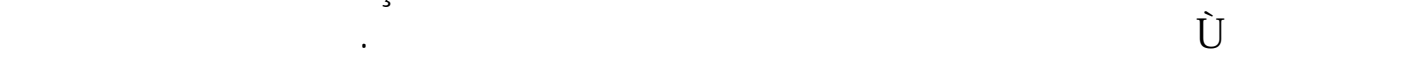

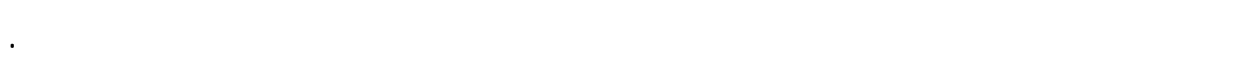

\section{THE EFFECT OF THE HEIGHT AND SPEED OF THE AIRPLANE CARRYING THE FOCUSED SYNTHETIC APERTURE RADAR ON THE AZIMUTH RESOLUTION}

\author{
Safwan Emad Abdul-Fatah and Prof. Khalil H. Sayidmarie \\ College of Electronic Eng. / University of Mosul / IRAQ
}

\begin{abstract}
:
Airborne imaging radar systems are known by their ability to produce high resolution images of ground targets using microwave region of electromagnetic waves. There are many military and civilian applications of imaging radar systems. The high resolution images achieved by transmitting a number of electromagnetic pulses to the earth surface then integrating them coherently at the receiver. A study have been achieved to show the effect of changing speed or height or both of the airplane carrying the focused synthetic aperture radar upon the azimuth resolution. The simulation results showed that increasing the speed or decreasing the height of the airplane will improve the azimuth resolution, and it can be noted for practical parameters that decreasing the speed and the height of the airplane leads to degraded azimuth resolution. The results of azimuth resolution obtained from simulations and those from theoretical calculations are nearly identical for targets of point reflectors.
\end{abstract}

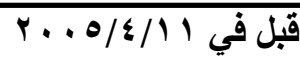

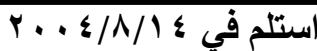


$\begin{array}{llll}\text { Al-Rafidain Engineering } \quad \text { Vol.13 No.4 } & 2005\end{array}$

Introduction:

:rPtoris1

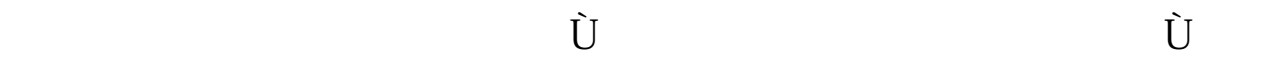

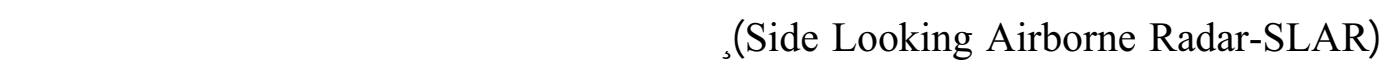

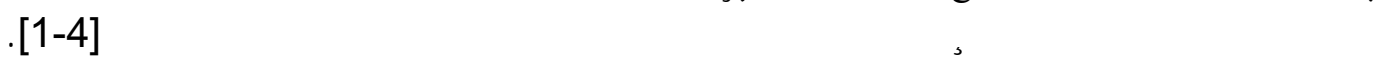
Gg (Real Aperture Radar-RAR) ry ry

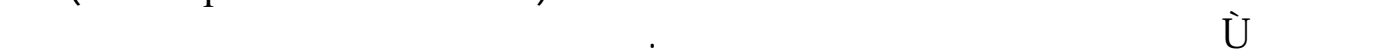

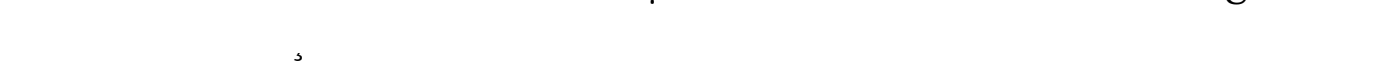

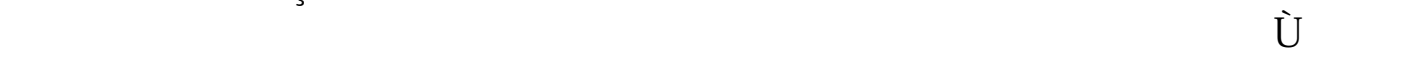

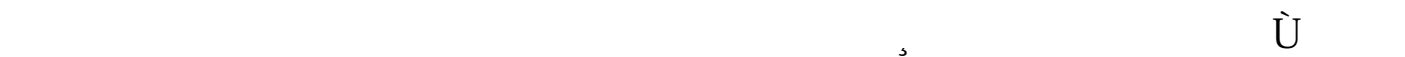

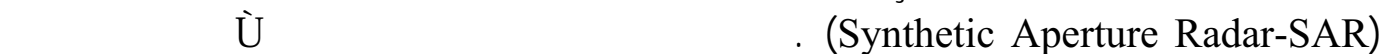
釉 ¿

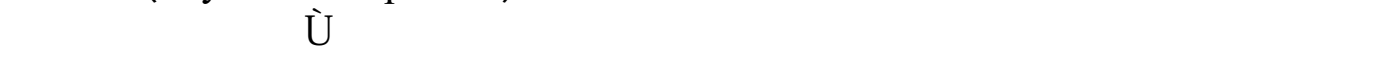

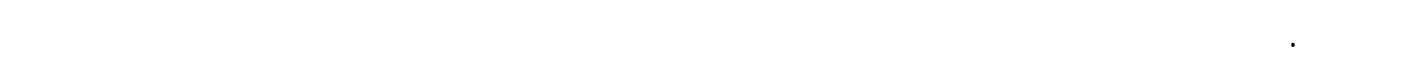

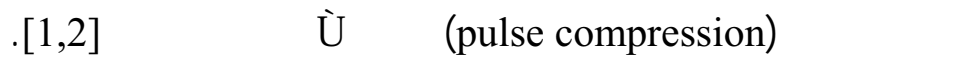

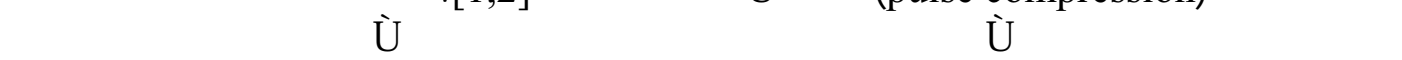

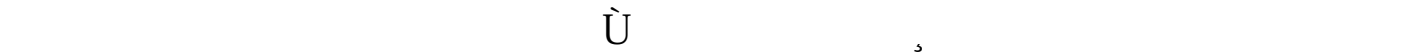

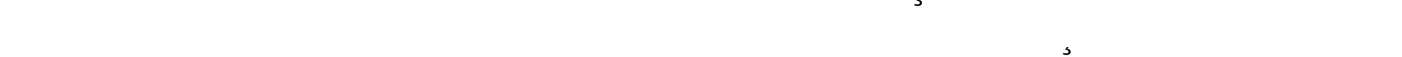

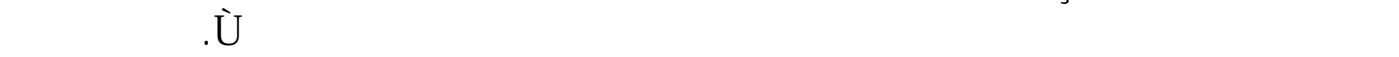

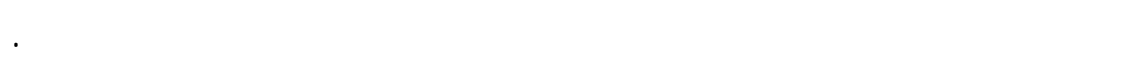

Azimuth Processing

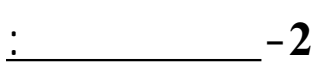

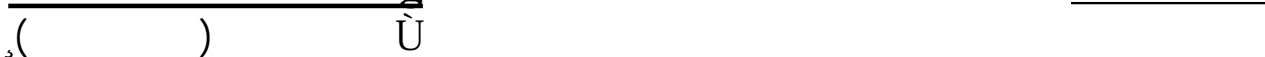

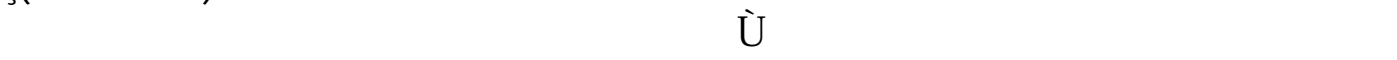

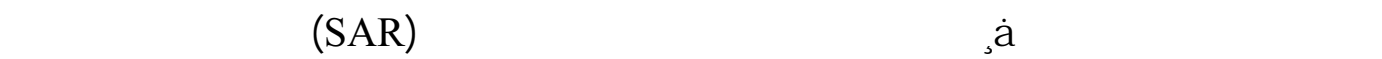

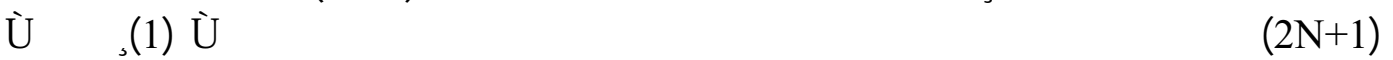

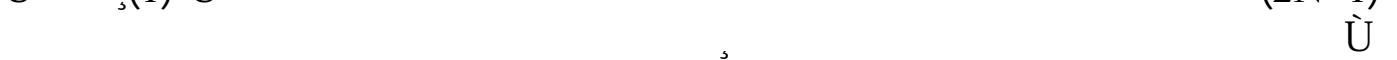

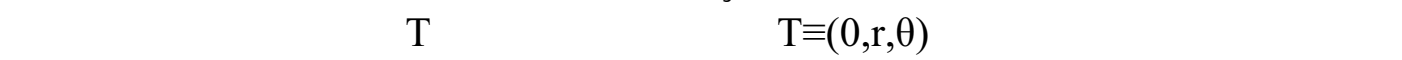

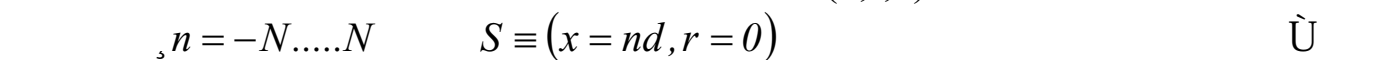

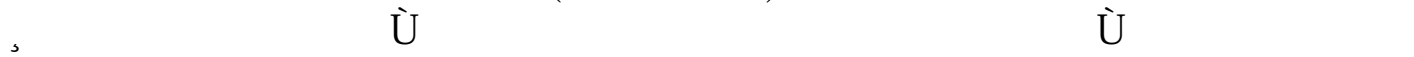

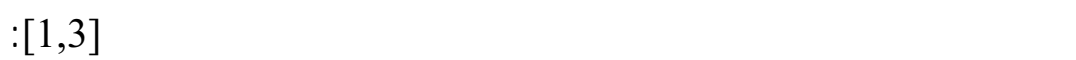

$$
L=\frac{\lambda r}{D}
$$

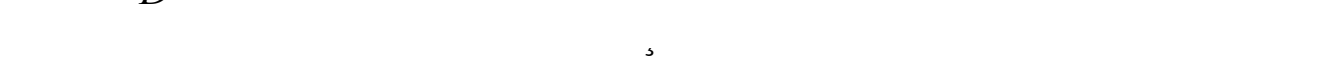

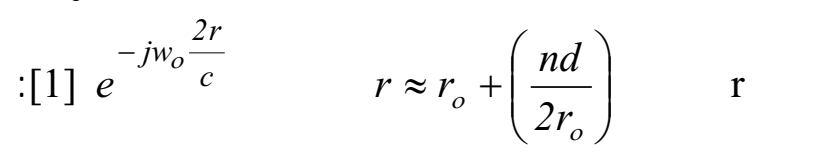

$$
\begin{aligned}
& f(n d)=e^{-j w_{o} \frac{2 r_{o}}{c}-j \frac{2 \pi}{\lambda r_{o}}(n d)^{2}} \\
& f(n d)=e^{-j \frac{2 \pi}{\lambda r_{o}}(n d)^{2}}
\end{aligned}
$$

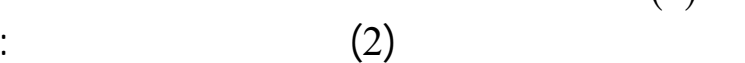


$\begin{array}{llll}\text { Al-Rafidain Engineering } \quad \text { Vol.13 No.4 } & 2005\end{array}$

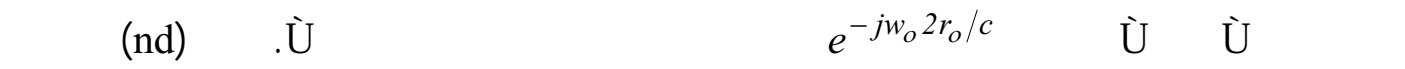

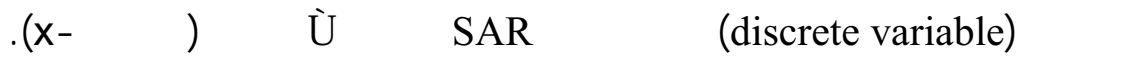

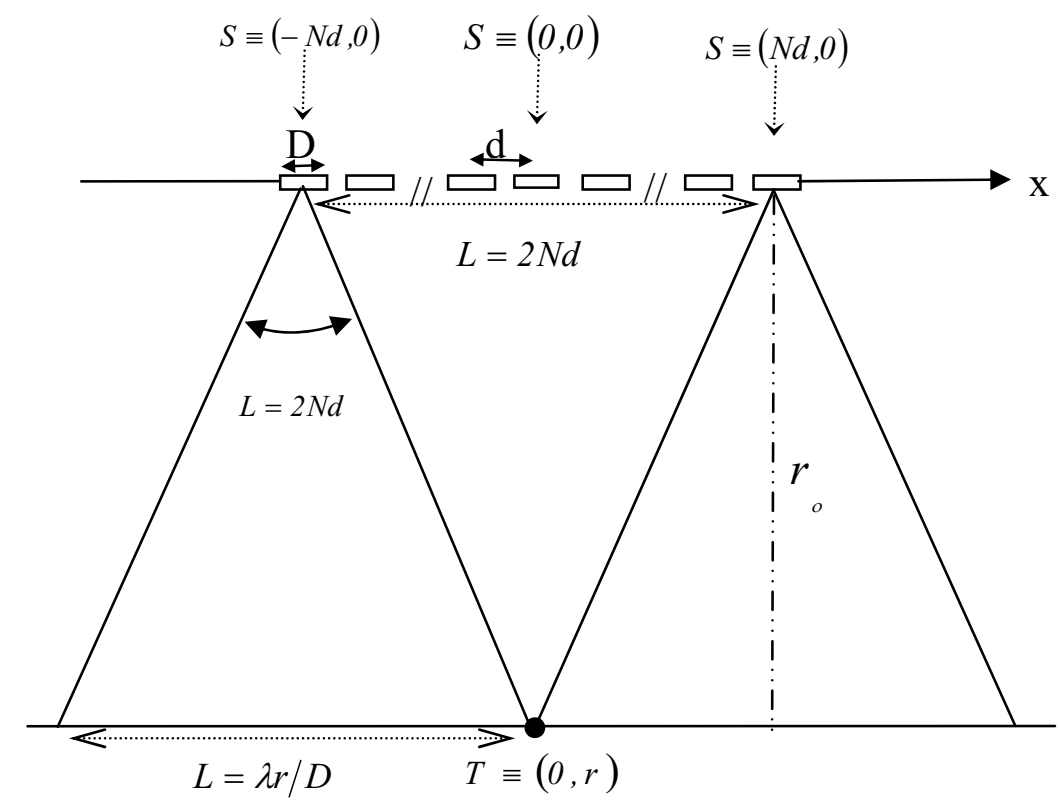

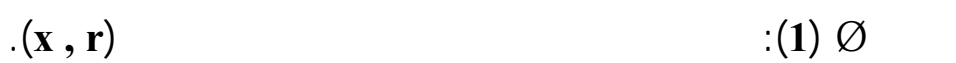

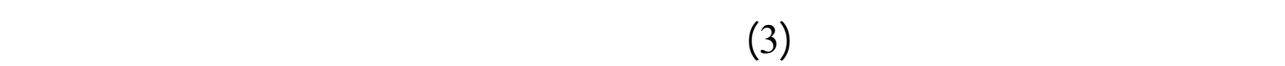

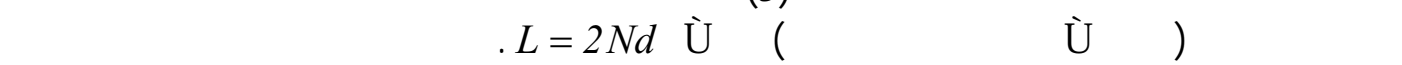

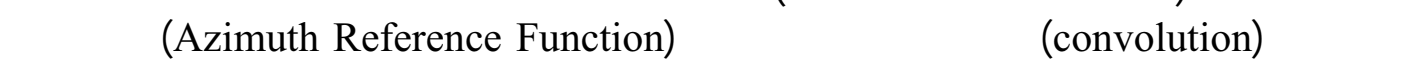

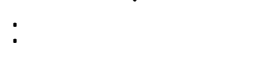
$g(n d)=e^{j \frac{2 \pi}{\lambda r_{o}}(n d)^{2}}$

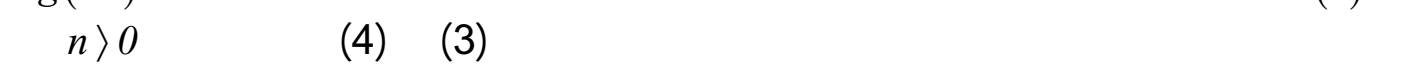

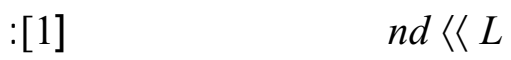

$$
f\left(x^{\prime}\right)=\frac{\sin \left(\frac{2 \pi L}{D} x^{\prime}\right)}{\frac{L}{d} \sin \left(\frac{2 \pi d}{D} x^{\prime}\right)}
$$

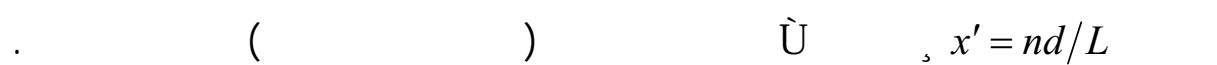

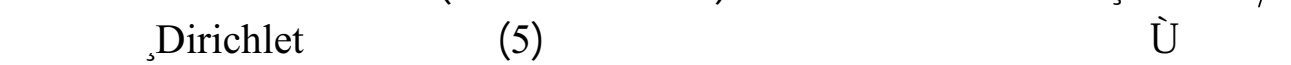

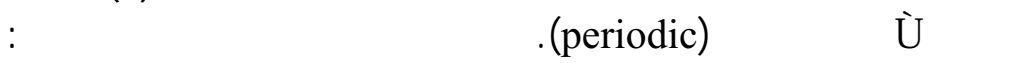

$$
2 \pi \frac{d}{D} x^{\prime}=q \pi \quad q=0, \pm 1, \pm 2, \ldots \ldots
$$


$\begin{array}{llll}\text { Al-Rafidain Engineering } \quad \text { Vol.13 No.4 } & 2005\end{array}$

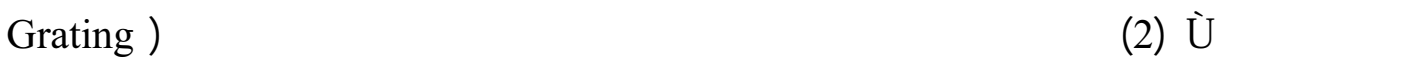

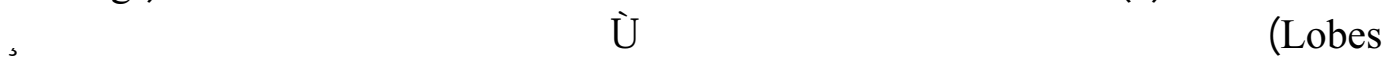

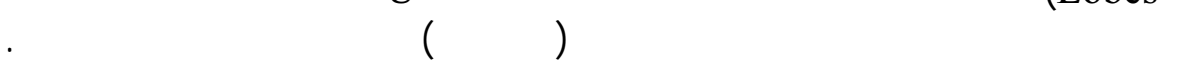

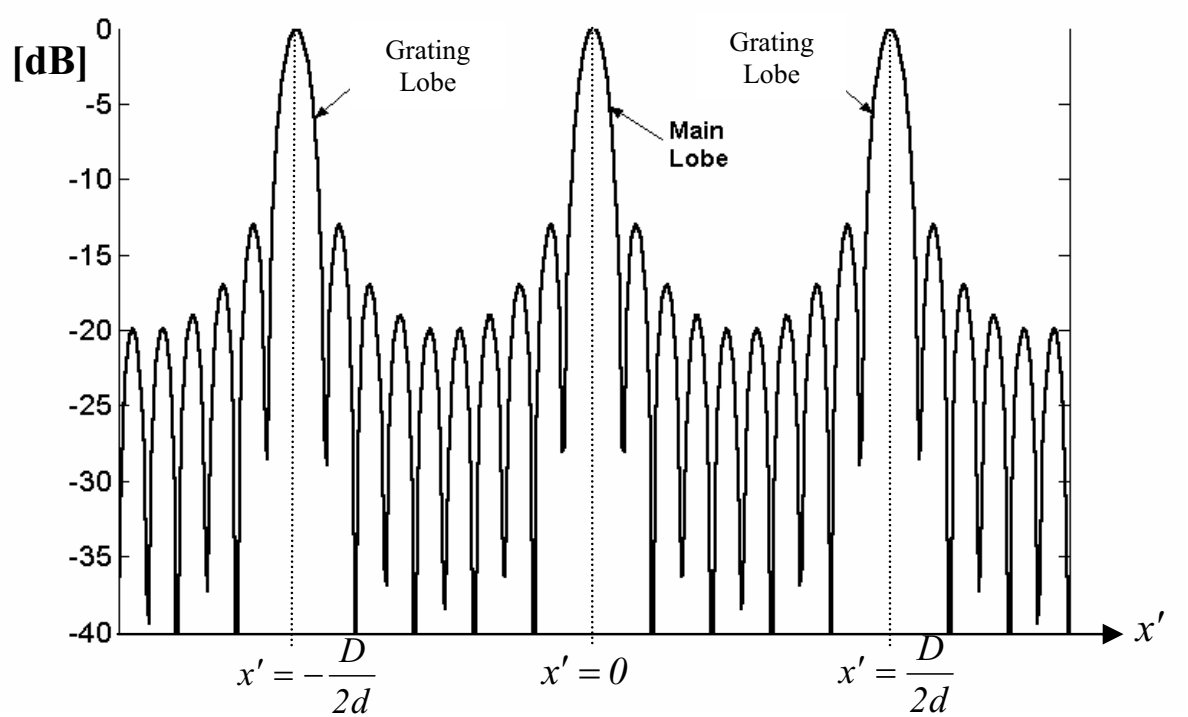

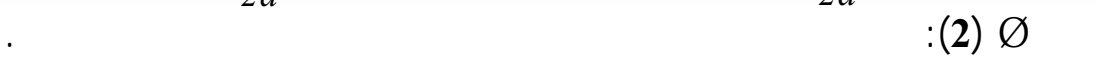

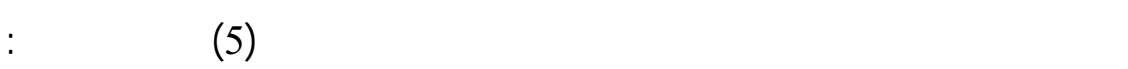

$$
2 \pi \frac{d}{D} \frac{1}{2} \leq \frac{\pi}{2} \quad \Rightarrow \quad \mathrm{d} \leq \frac{\mathrm{D}}{2}
$$

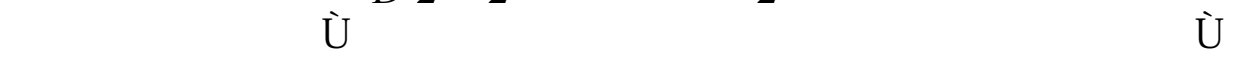

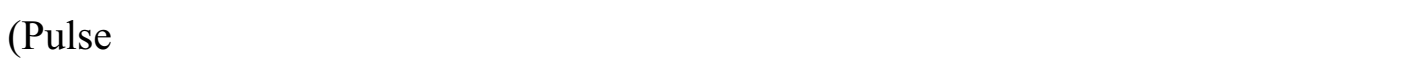

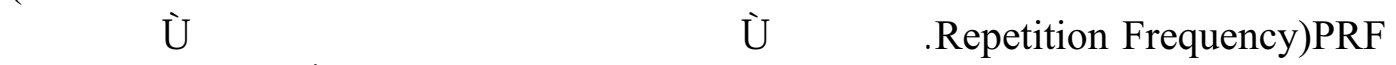

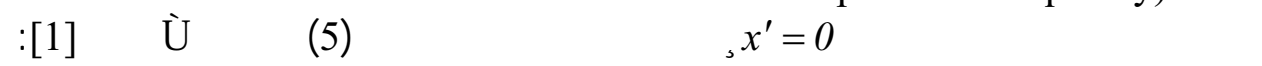
$f\left(x^{\prime}\right)=\sin c\left(\frac{2 \pi L}{D} x^{\prime}\right)$

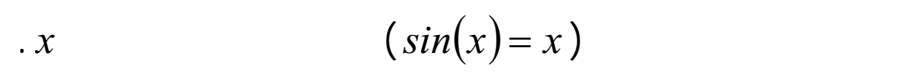

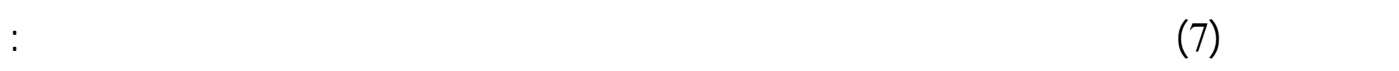

$$
\rho_{x}^{\prime}=\frac{D}{2 L}
$$

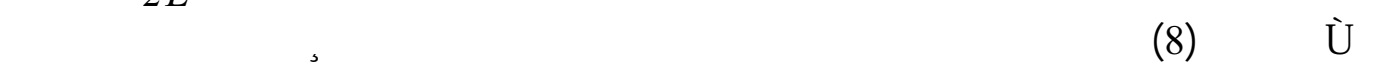

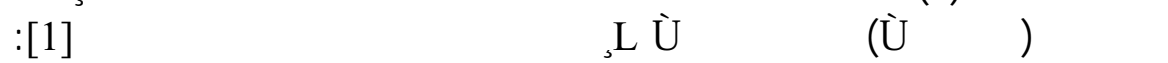

$$
\rho_{x}=\frac{D}{2}
$$

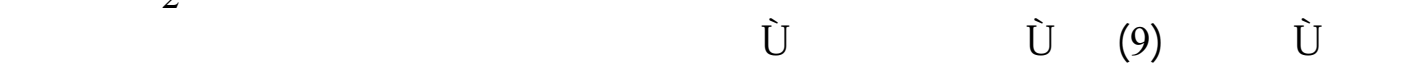

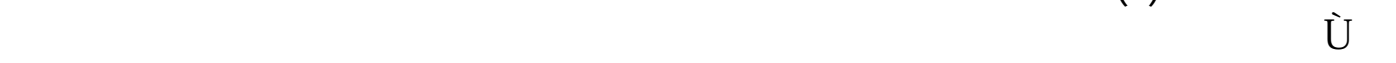

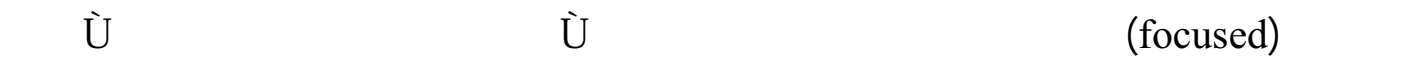


$\begin{array}{llll}\text { Al-Rafidain Engineering } \quad \text { Vol.13 No.4 } & 2005\end{array}$

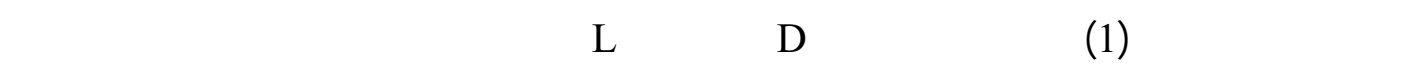

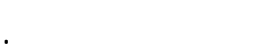

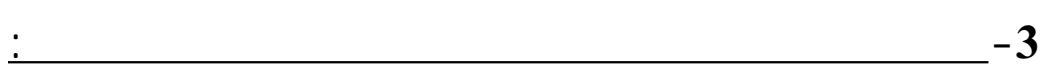

Doppler Variation with Phase and Frequency History of a Point

Return:

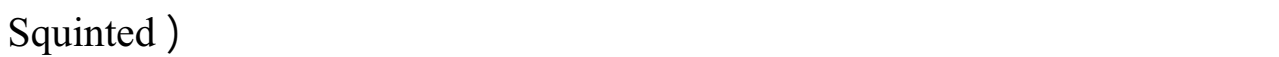

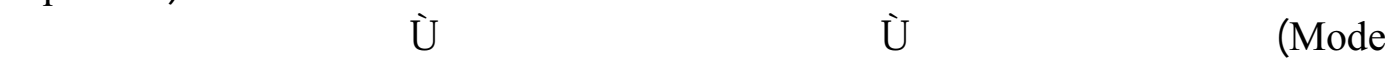

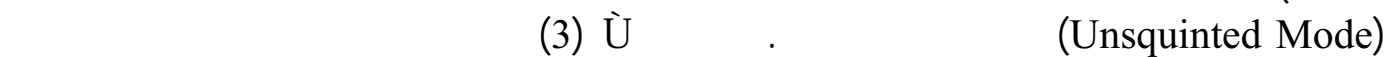

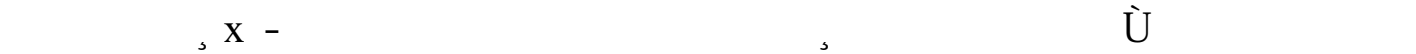

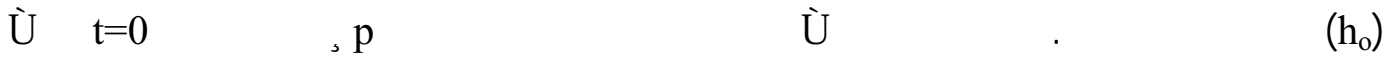

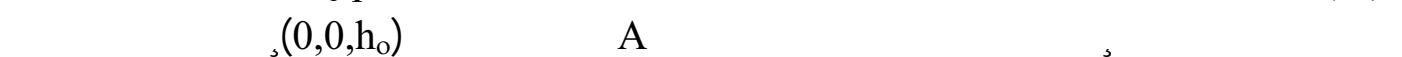

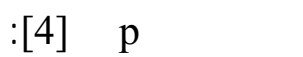

$$
r_{o}=\left(x_{o}^{2}+y_{o}^{2}+h_{o}^{2}\right)^{\frac{1}{2}}
$$

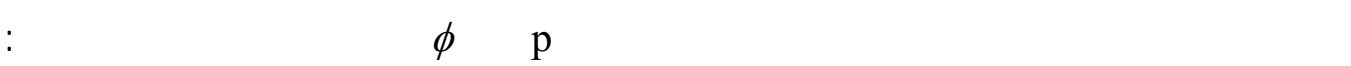
$\cos \phi=\frac{x_{o}}{r_{o}}$

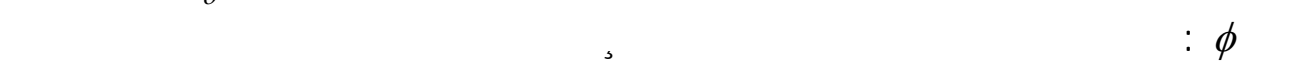

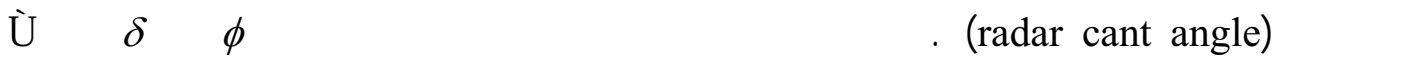

$$
\cos \phi=\cos \delta \cos \psi
$$

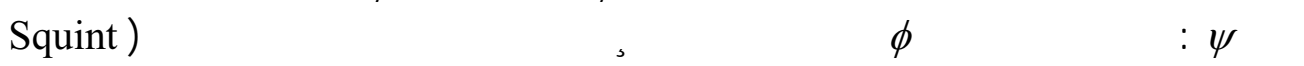

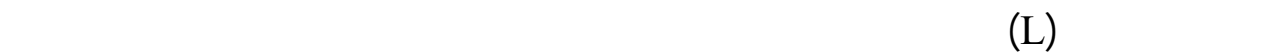
(Angle

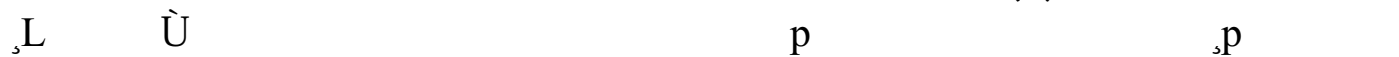

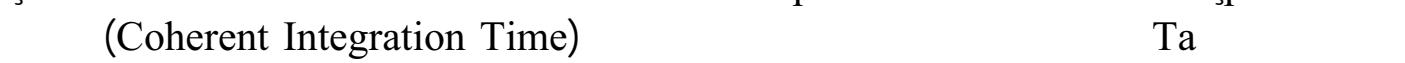

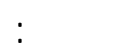

$$
T_{a}=\frac{L}{v}
$$

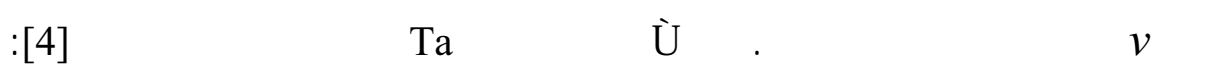

$$
r(t)=\left[y^{2}+\left(x_{o}-v t\right)^{2}+h^{2}\right]^{\frac{1}{2}} \quad-T_{a} / 2 \leq t \leq T_{a} / 2
$$

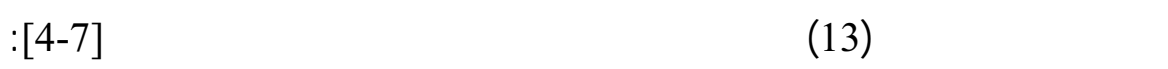




$$
r(t)=r_{o}-v t \cos \phi+\frac{v^{2} t^{2}}{2 r_{o}} \sin ^{2} \phi
$$

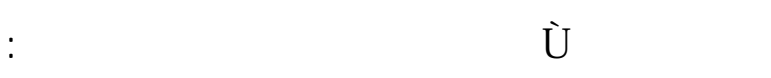

$$
f_{d}=\frac{2}{\lambda} \frac{\partial r(t)}{\partial t}
$$

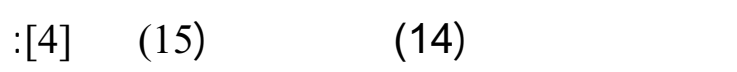

$$
f_{d}=\frac{2 v}{\lambda} \cos \phi-\frac{2 v^{2} t}{\lambda r_{o}} \sin ^{2} \phi \quad-\frac{\mathrm{T}_{\mathrm{a}}}{2} \leq t \leq \frac{T_{a}}{2}
$$

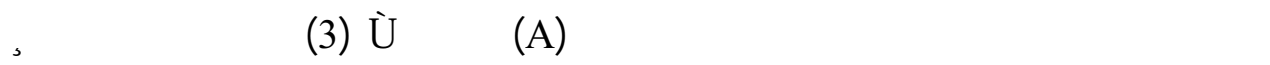

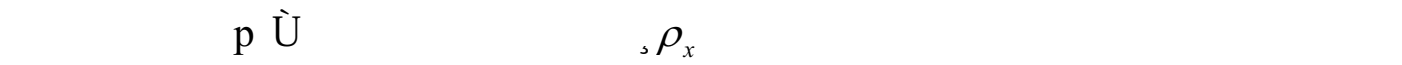

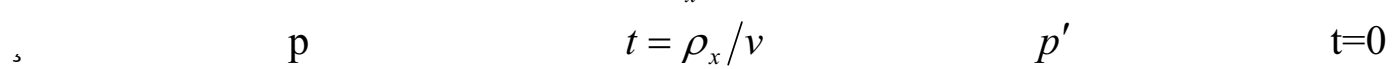

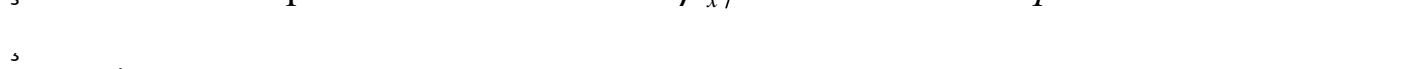

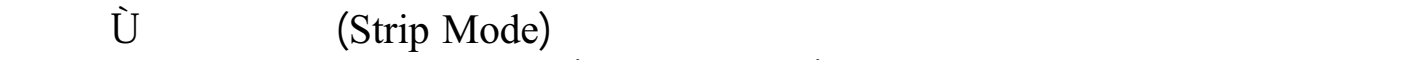

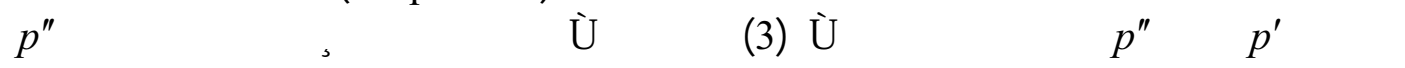

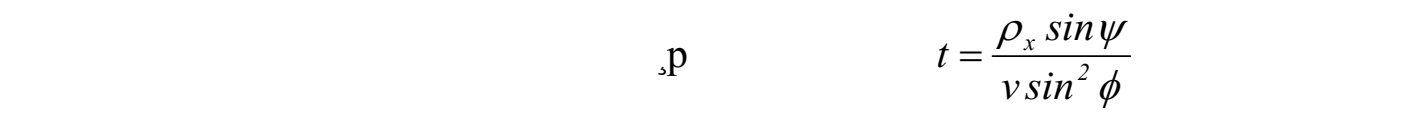

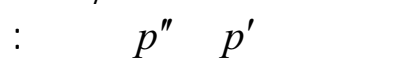

$$
\begin{gathered}
\Delta f_{d}=\left.f_{d}\right|_{t=0}-\left.f_{d}\right|_{t=\frac{\rho_{x} \sin \psi}{v \sin ^{2} \phi}} \\
\Delta f_{d}=\frac{2 v}{\lambda r_{o}} \rho_{x} \sin \psi
\end{gathered}
$$

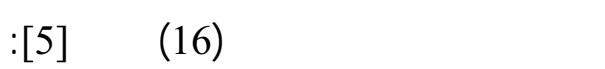

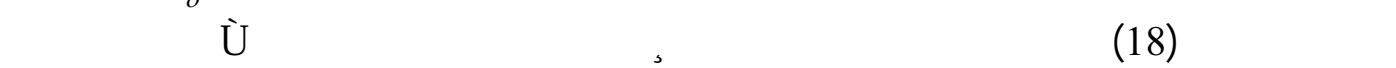

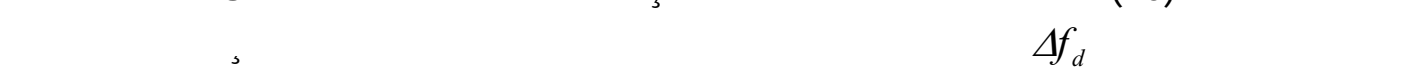

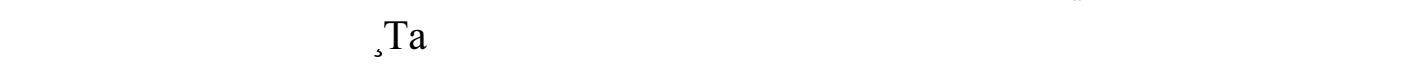

$$
\begin{gathered}
\Delta f_{d}=\frac{1}{T_{a}}=\frac{v}{L} \\
\rho_{x}=\frac{\lambda r_{o}}{2 L} \csc \psi
\end{gathered}
$$

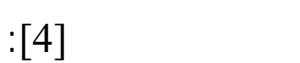

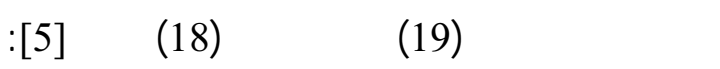

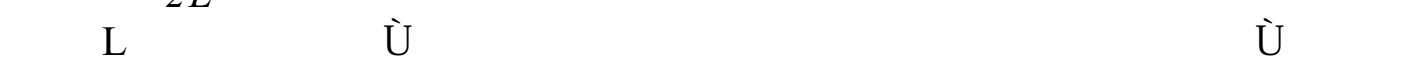

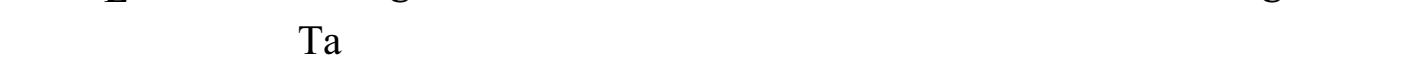


Al-Rafidain Engineering $\quad$ Vol.13 No.4 2005

$\Delta f_{d}=\frac{1}{T_{a \max }}=\frac{v}{L_{\max }}$

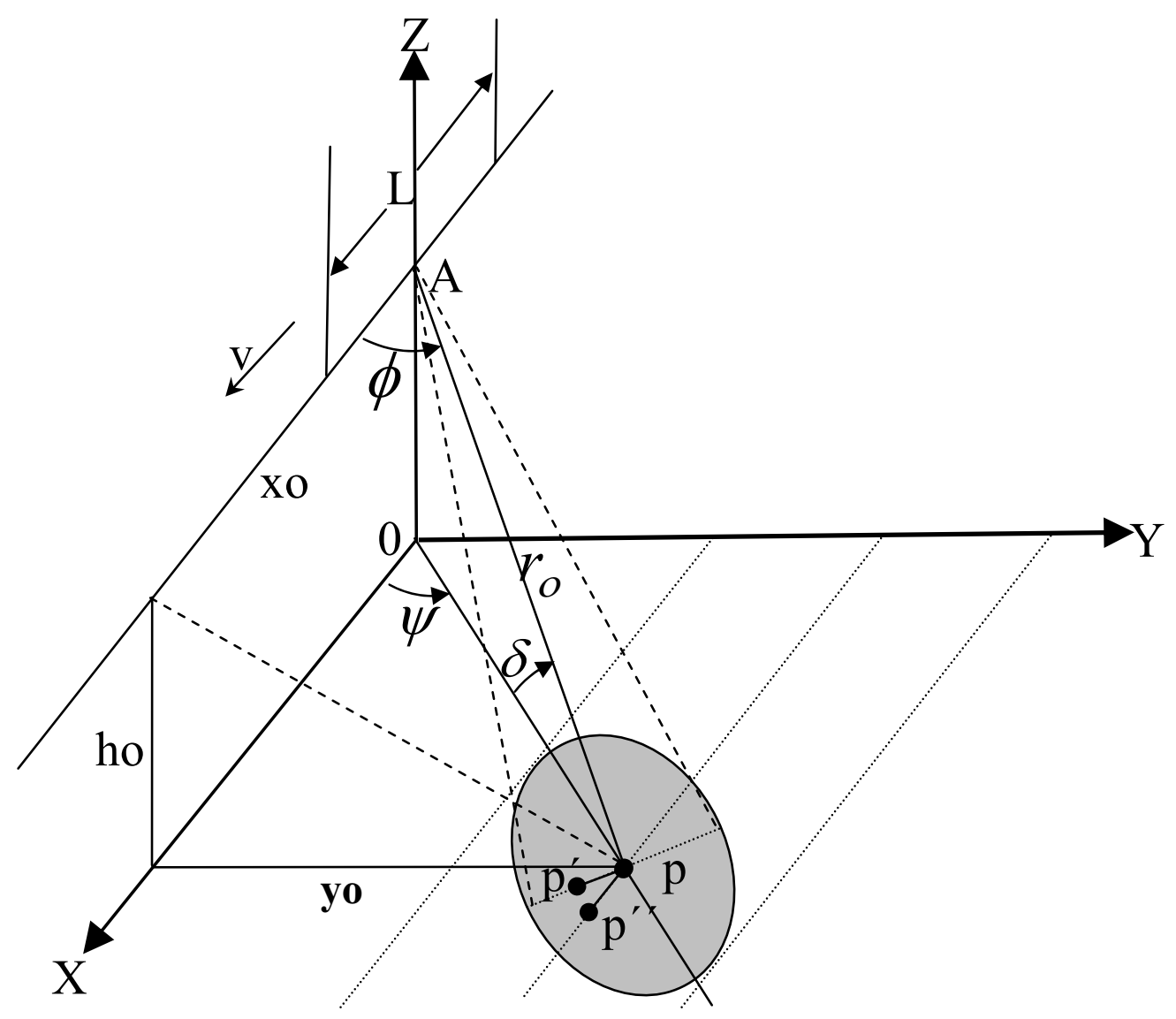

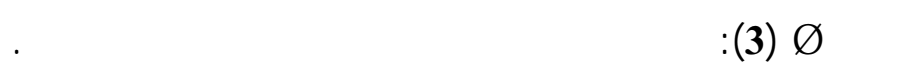

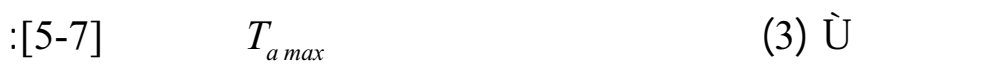

$$
T_{a \max }=\frac{r_{o} \phi_{a}}{v \gamma_{g}}
$$

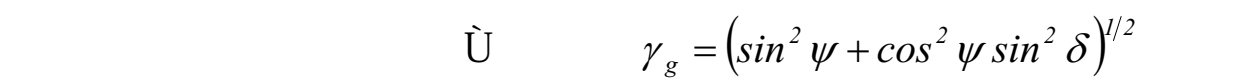

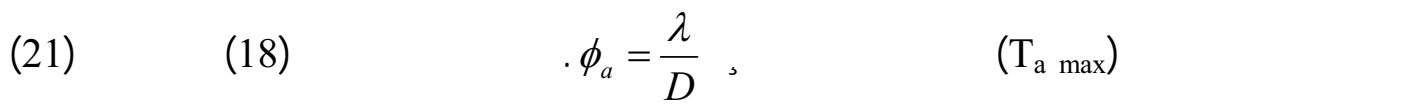
$\rho_{x}=\frac{D}{2} \sqrt{1+\cot ^{2} \psi \sin ^{2} \delta}$ 
$\begin{array}{llll}\text { Al-Rafidain Engineering } \quad \text { Vol.13 No.4 } & 2005\end{array}$

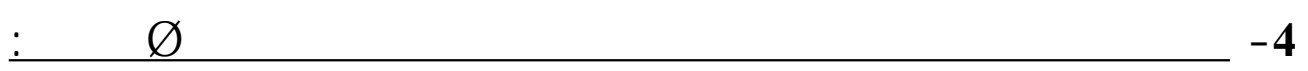

Performance evaluation for the focused SAR system under

various operating conditions:

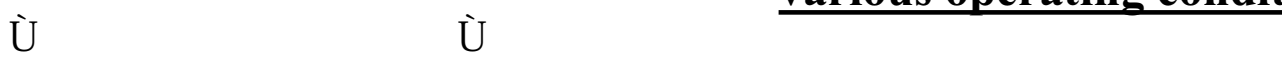

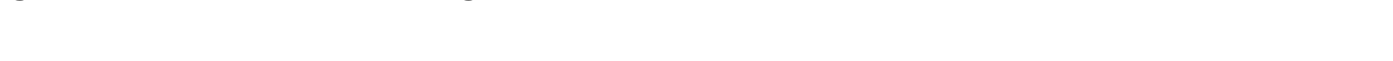

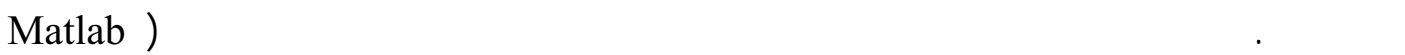

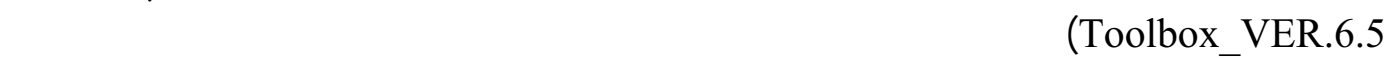

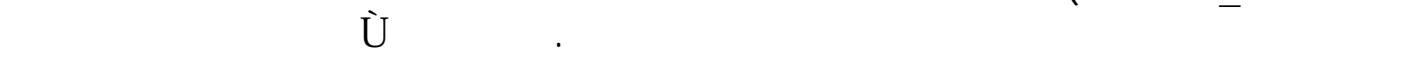

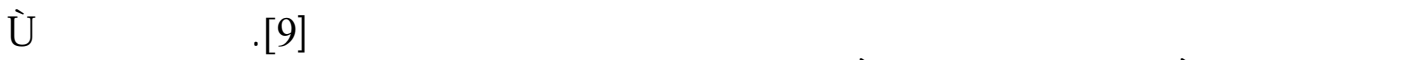

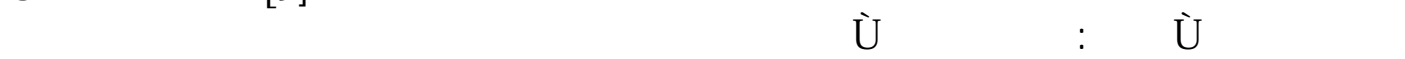

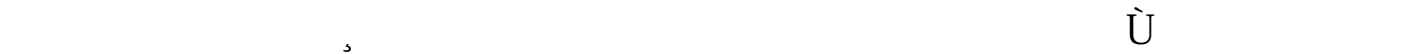

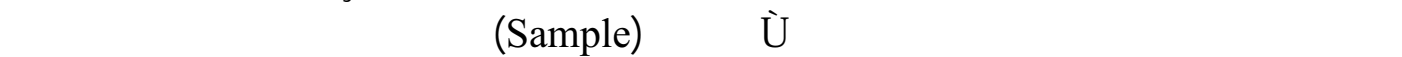

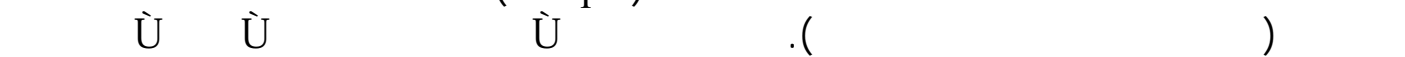

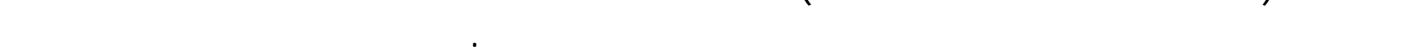

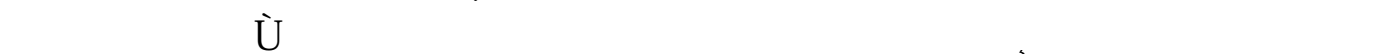

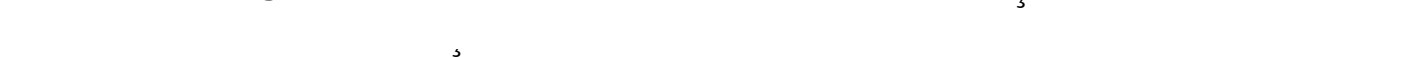
ŶCŠ r ry

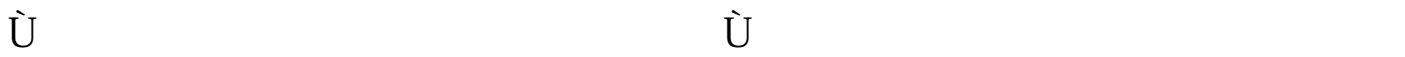

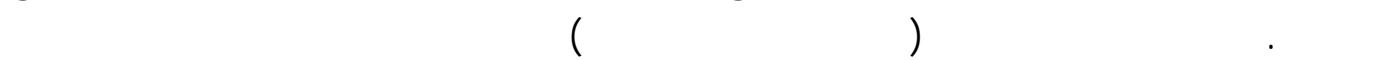

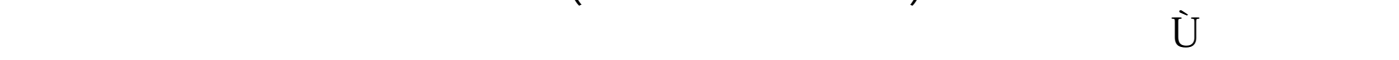

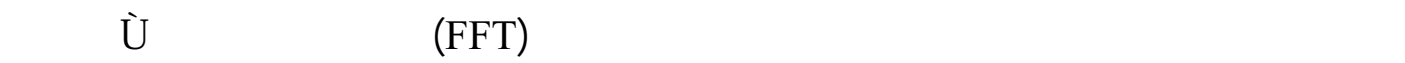

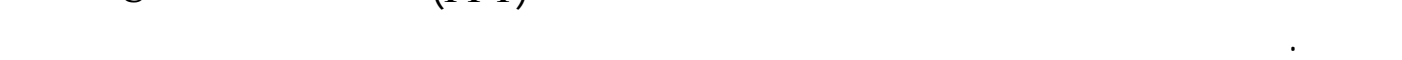

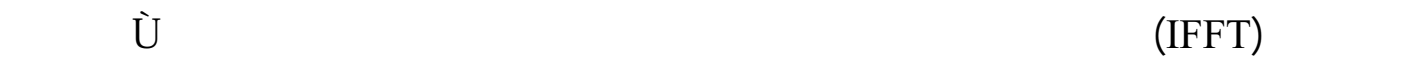

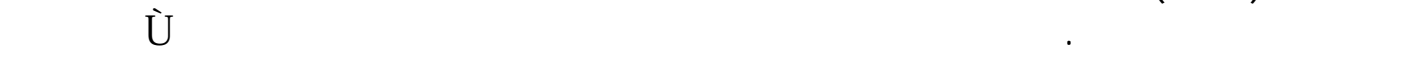

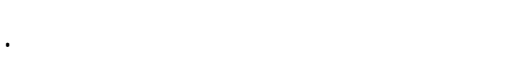

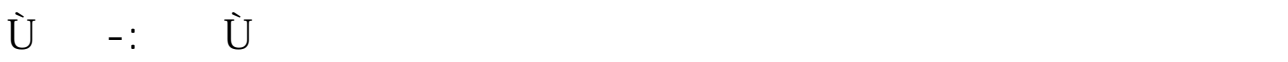

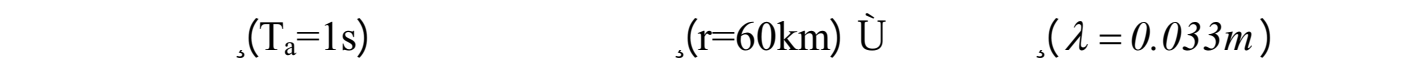

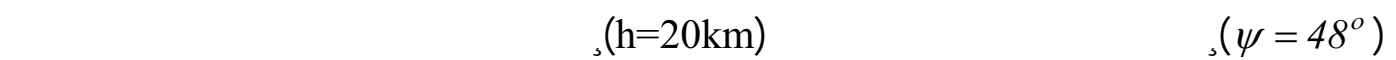

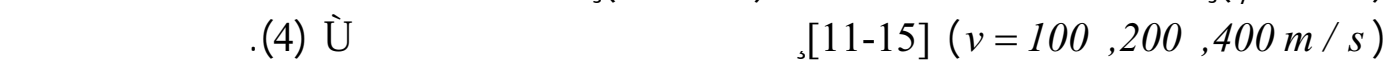

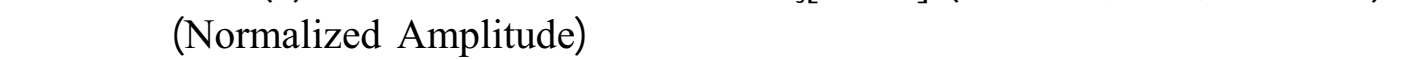

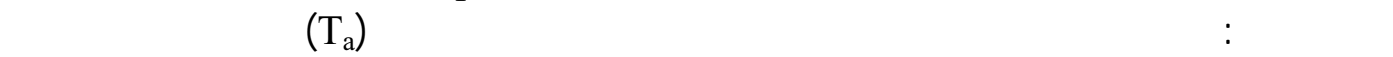

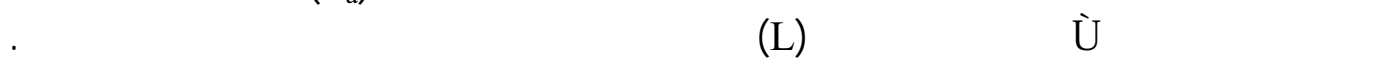

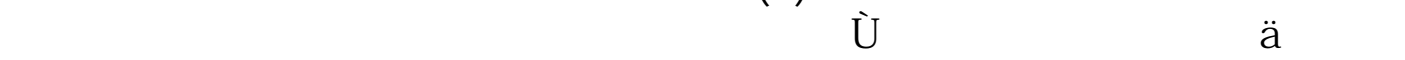

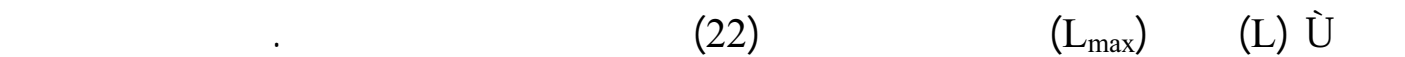

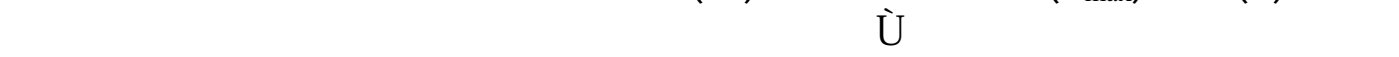

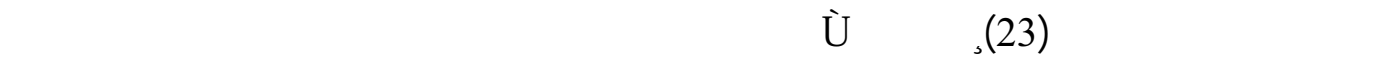

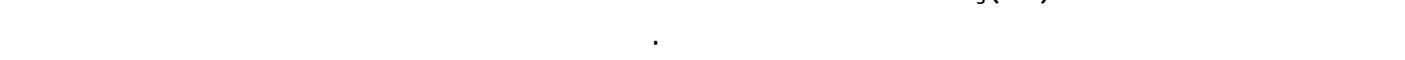

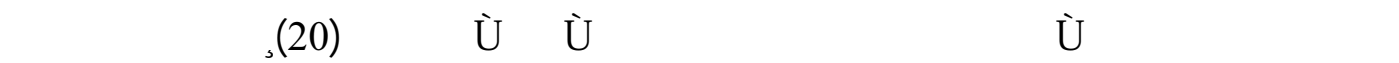

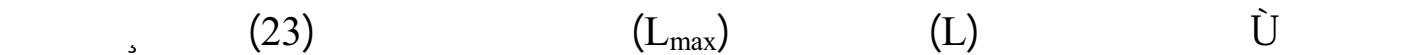

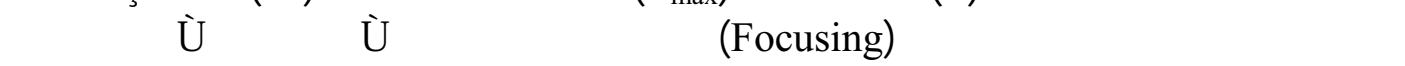

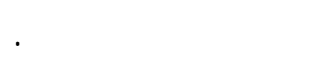


$\begin{array}{llll}\text { Al-Rafidain Engineering } \quad \text { Vol.13 No.4 } & 2005\end{array}$
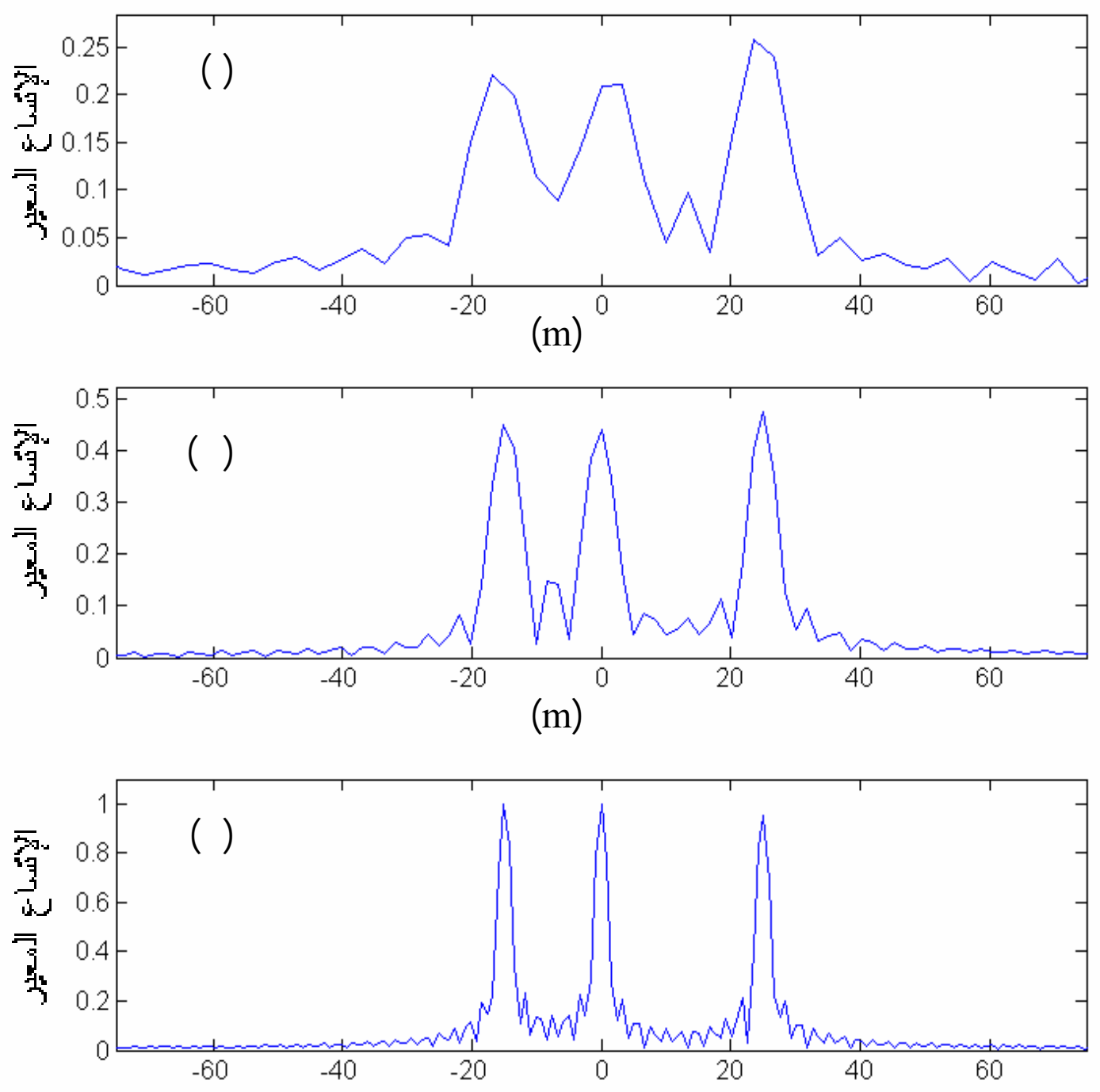

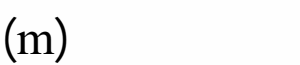

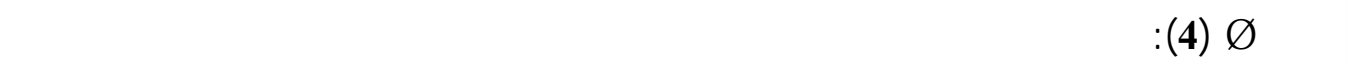

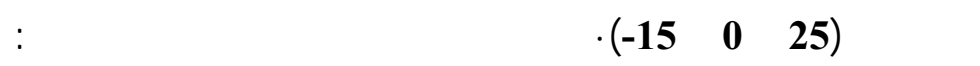
$v=400 \mathrm{~m} / \mathrm{s} \mathbf{- \mathbf { S }}$
$v=200 m / s-\mathbf{R}$
$v=100 m / s-\check{\mathbf{n}}$

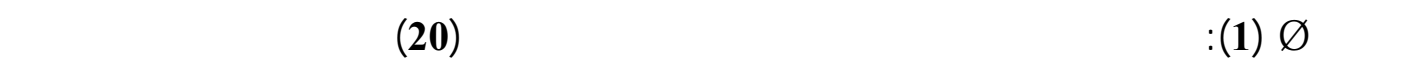
r.

\begin{tabular}{|c|c|c|c|}
\hline & \multicolumn{2}{|c|}{ 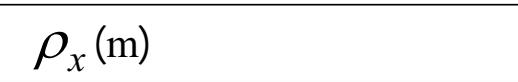 } & \multirow[b]{2}{*}{ 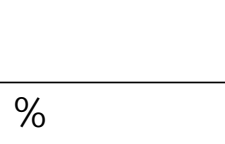 } \\
\hline$v(m / s)$ řŷũŭḱ́ & 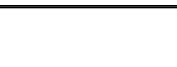 & 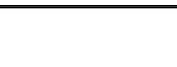 & \\
\hline |ิ̂ิ| & 13.4 & 13.5 & $0.75 \%$ \\
\hline छा & 6.7 & 7.3 & $8.95 \%$ \\
\hline â & 3.35 & 3.7 & $10.44 \%$ \\
\hline
\end{tabular}


$\begin{array}{llll}\text { Al-Rafidain Engineering } \quad \text { Vol.13 No.4 } & 2005\end{array}$

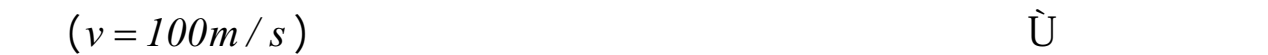

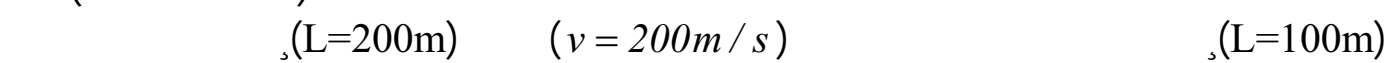

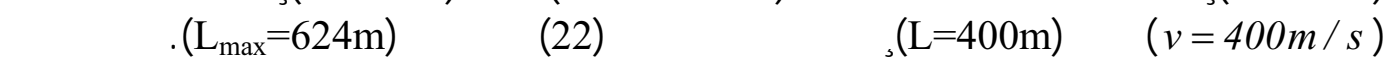

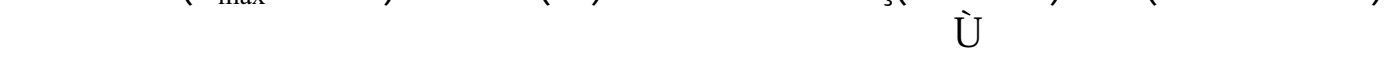
Đy

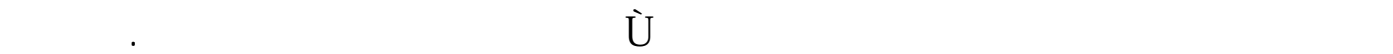
Gž řt Ų

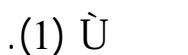

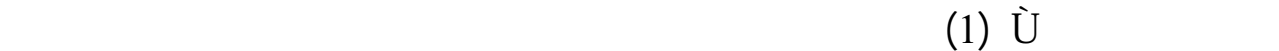

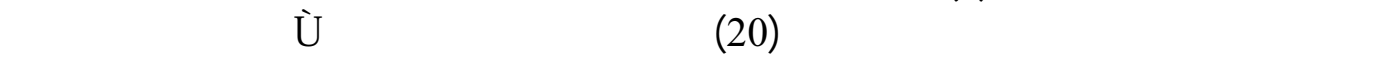

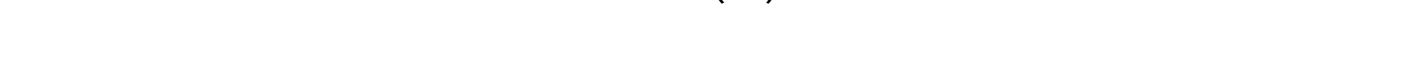

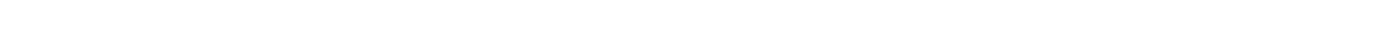

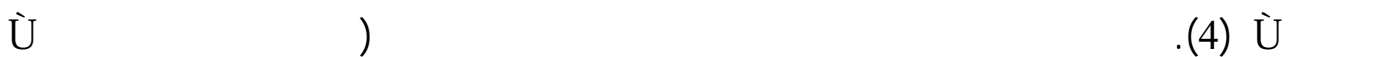

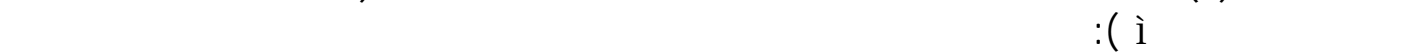

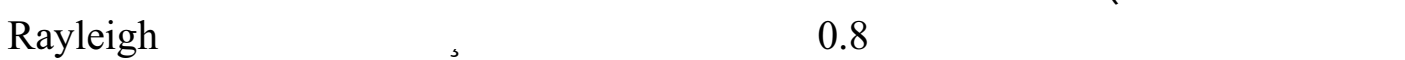

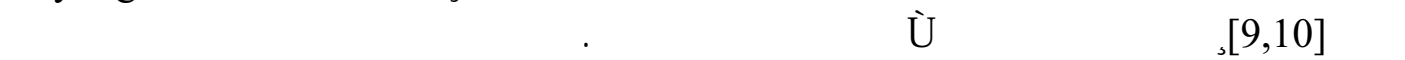

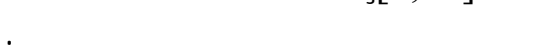

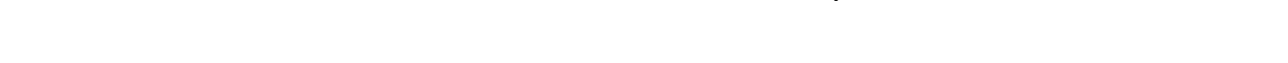

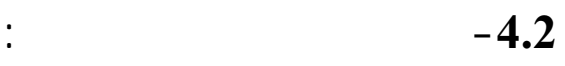

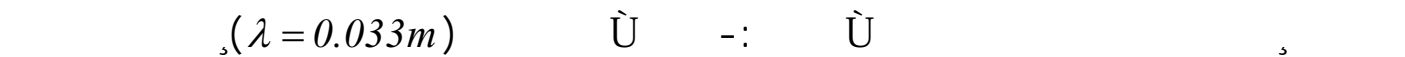

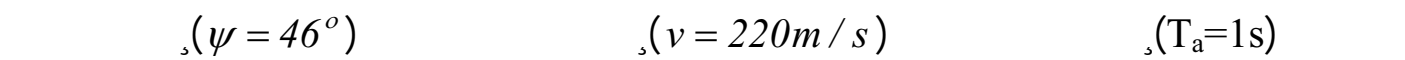

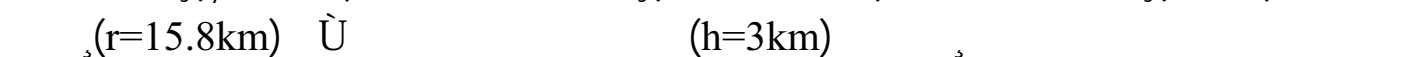

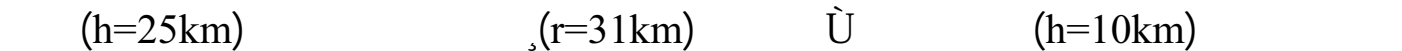

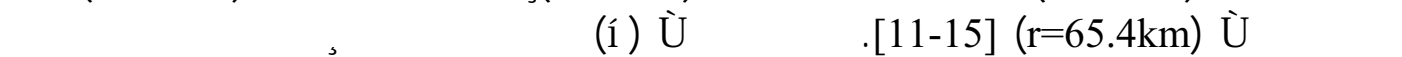

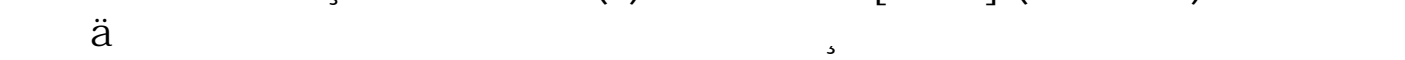

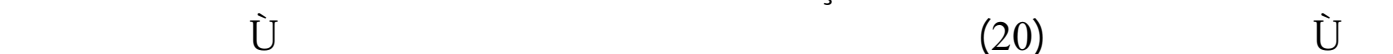

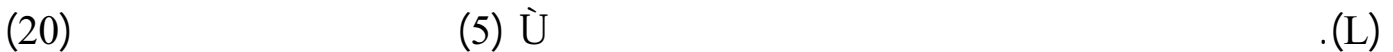

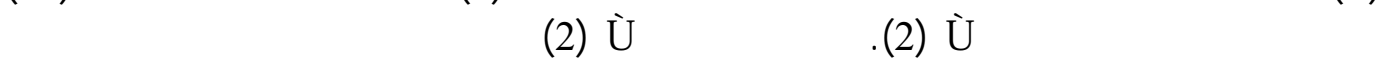

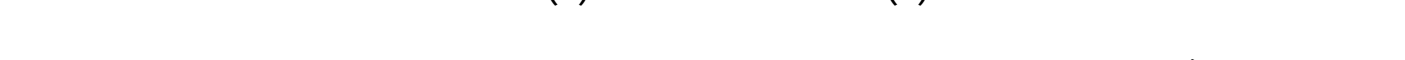

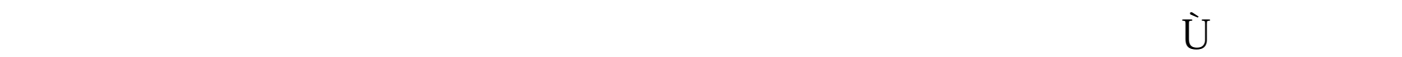

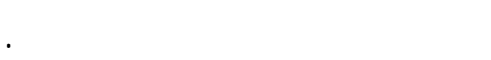

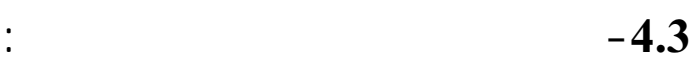

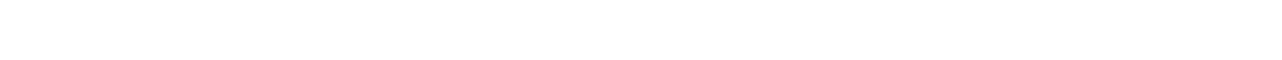

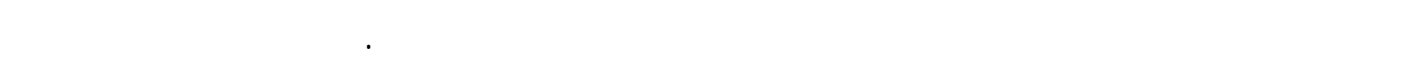
Đây̆

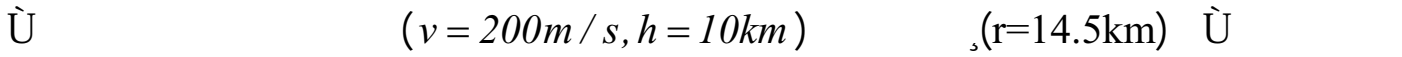

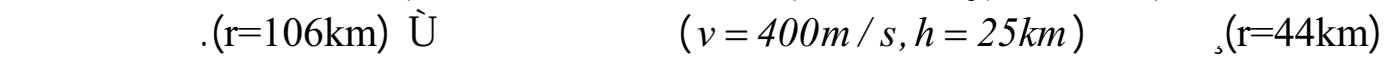

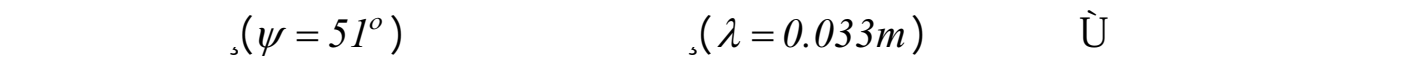

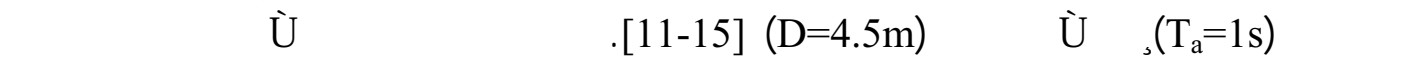

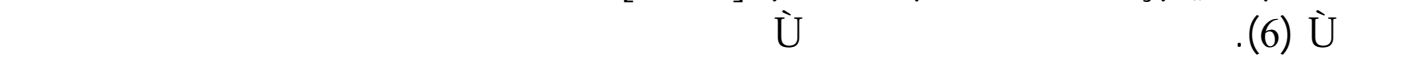

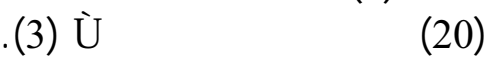


$\begin{array}{llll}\text { Al-Rafidain Engineering } \quad \text { Vol.13 No.4 } & 2005\end{array}$

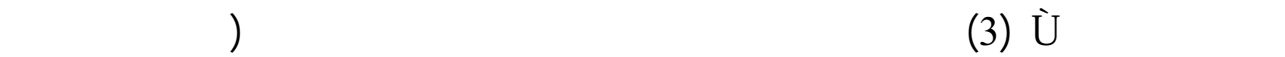

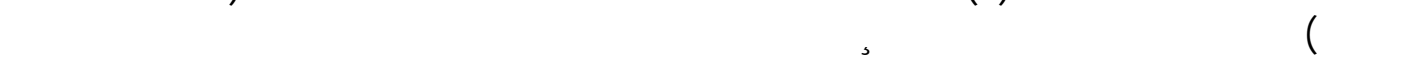

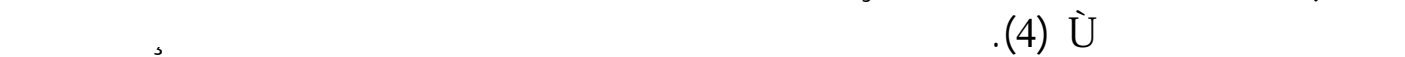

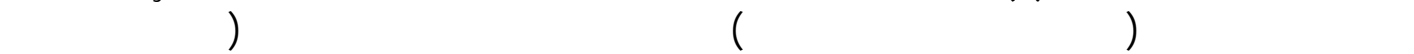

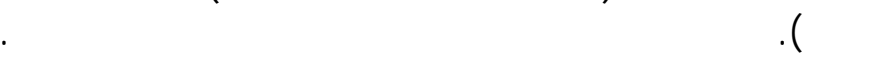

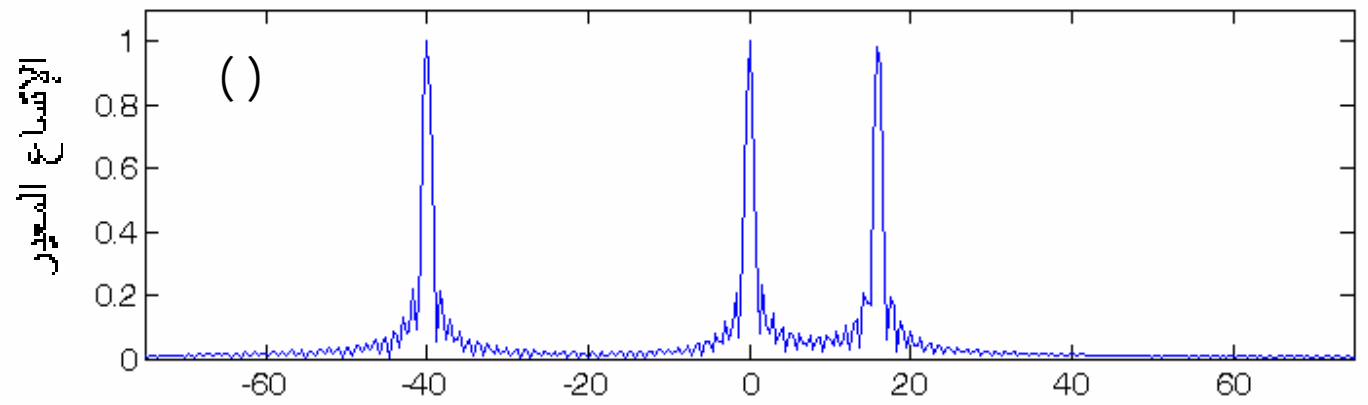

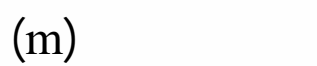

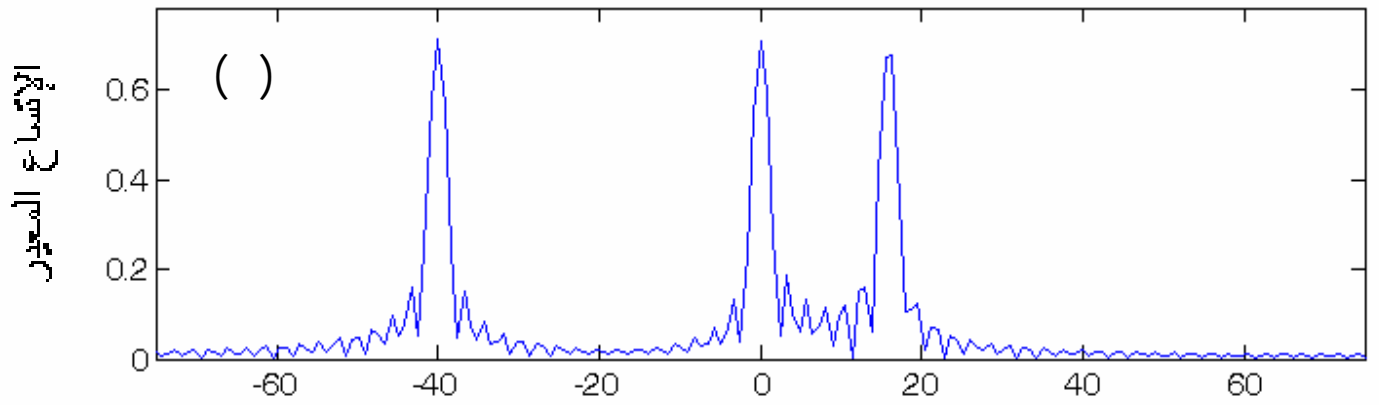

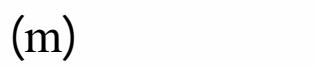

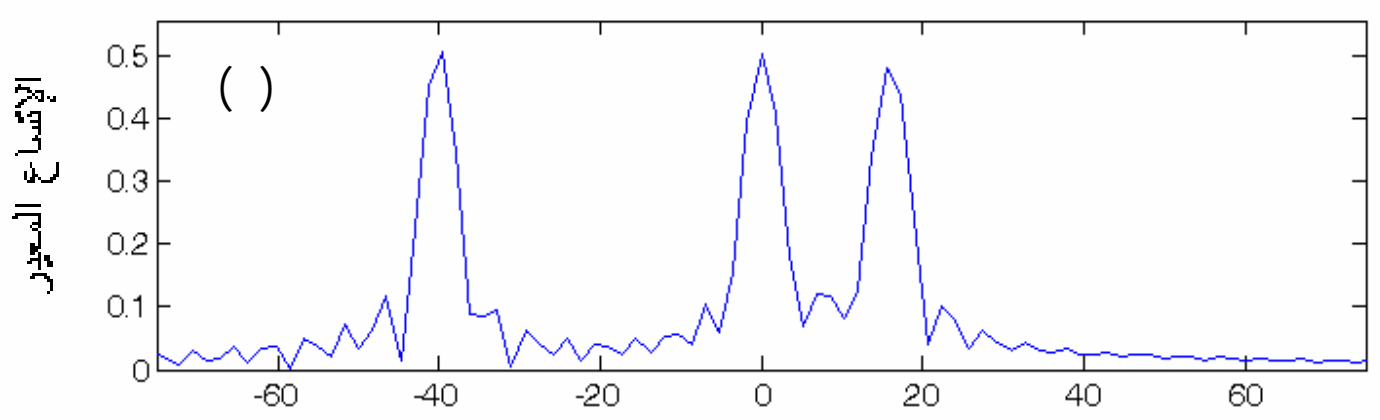

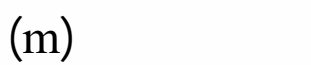

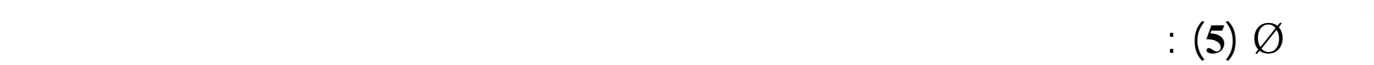

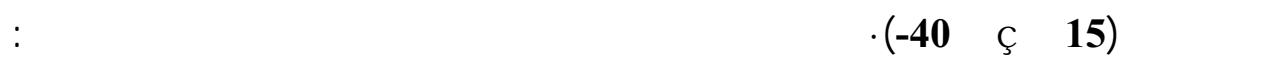

$$
h=25 \mathrm{~km}-\mathbf{S}
$$$$
h=10 \mathrm{~km}-\mathbf{R}
$$$$
h=3 \mathrm{~km}-\check{\mathbf{n}}
$$ 


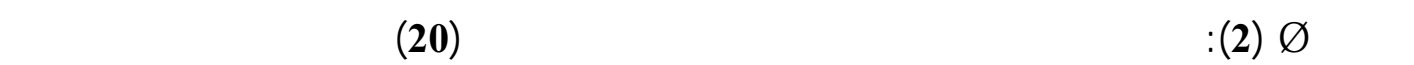

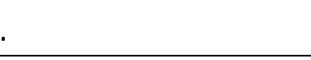

\begin{tabular}{|c|c|c|c|}
\hline & \multicolumn{2}{|c|}{ 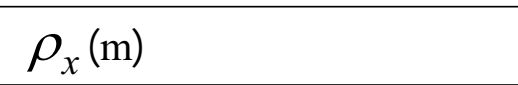 } & \\
\hline 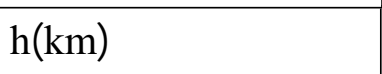 & 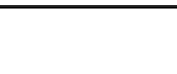 & 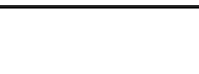 & 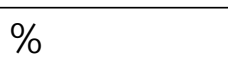 \\
\hline 3 & 1.7 & 1.9 & $11.76 \%$ \\
\hline 10 & 3.3 & 3.4 & $3.03 \%$ \\
\hline 25 & 6.9 & 7 & $1.45 \%$ \\
\hline
\end{tabular}

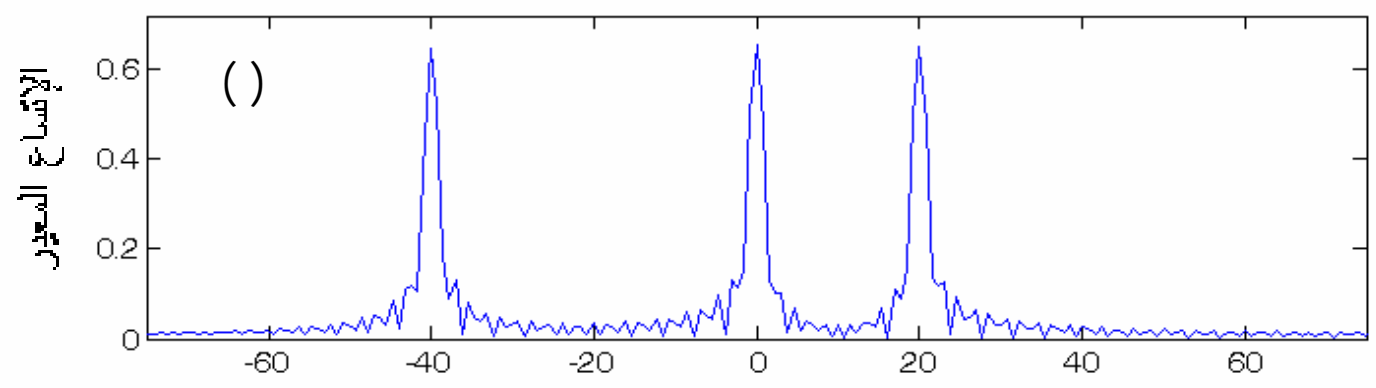

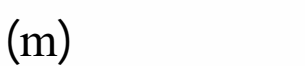

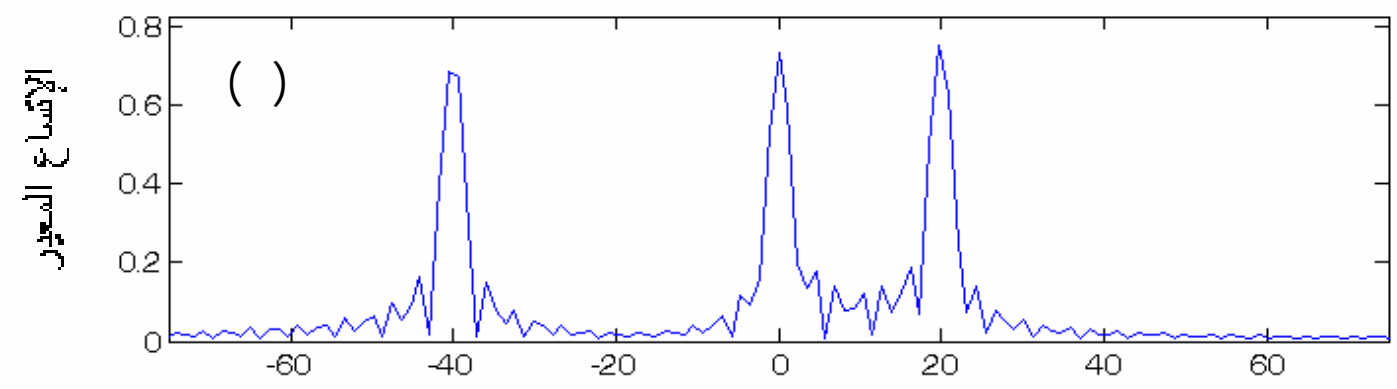

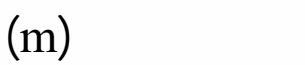

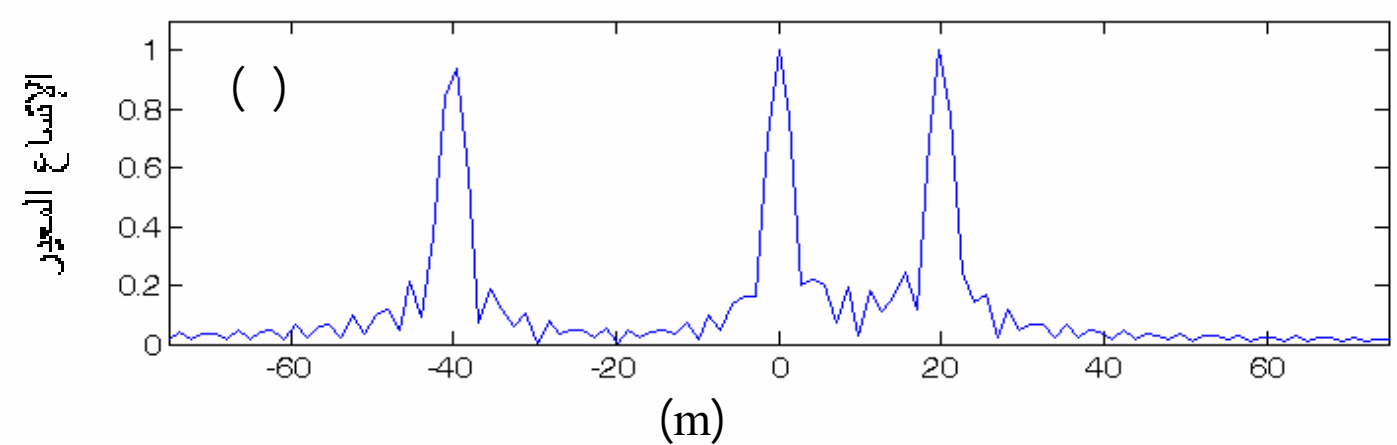

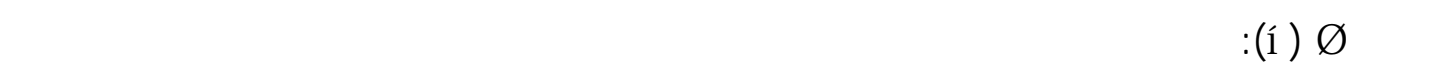

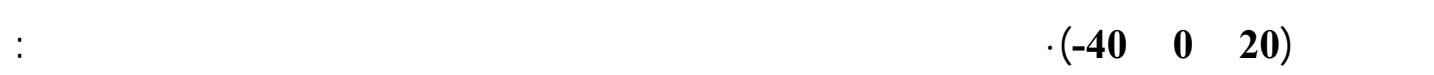
$v=400 \mathrm{~m} / \mathrm{s}, h=25 \mathrm{~km}-\mathbf{\mathbf { S }} \quad v=200 \mathrm{~m} / \mathrm{s}, h=10 \mathrm{~km}-\mathbf{R} \quad v=100 \mathrm{~m} / \mathrm{s}, h=3 \mathrm{~km}-\check{\mathbf{n}}$ 
$\begin{array}{llll}\text { Al-Rafidain Engineering } \quad \text { Vol.13 No.4 } & 2005\end{array}$

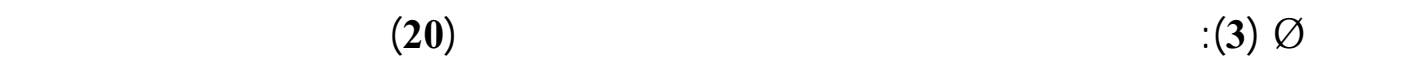

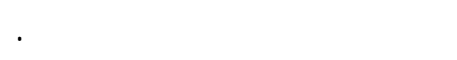

\begin{tabular}{|c|c|c|c|c|}
\hline \multirow[b]{2}{*}{ 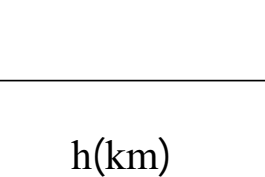 } & \multirow[b]{2}{*}{$v(m / s)$ řŷũǔt́́ } & \multicolumn{2}{|c|}{ 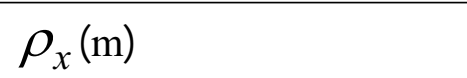 } & \multirow[b]{2}{*}{ 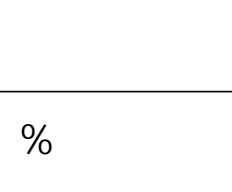 } \\
\hline & & řler̈YdB́̉C & 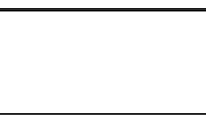 & \\
\hline 3 & 100 & 3 & 3.1 & $3.33 \%$ \\
\hline 10 & 200 & 4.6 & 4.7 & $2.17 \%$ \\
\hline 25 & 400 & 5.6 & 5.7 & $1.78 \%$ \\
\hline
\end{tabular}

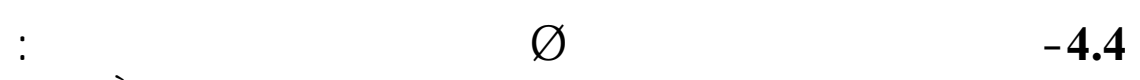

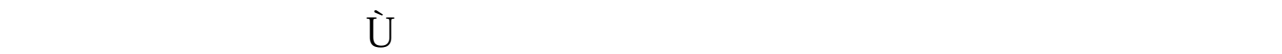

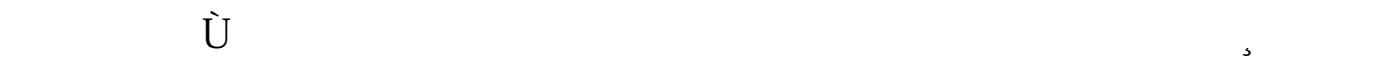

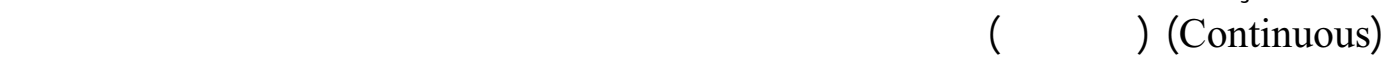

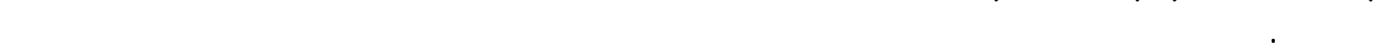

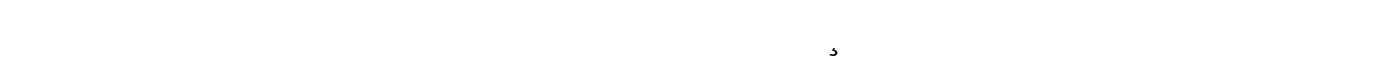

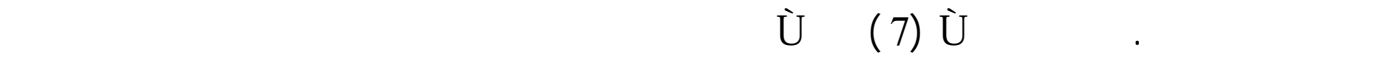

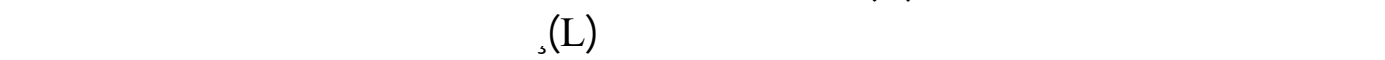

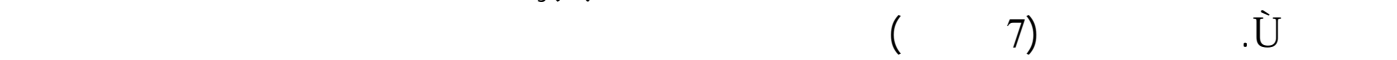

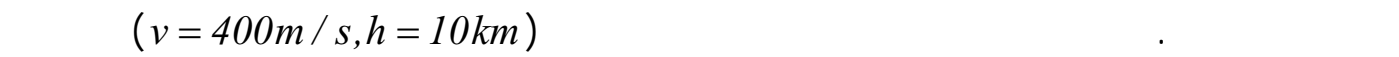

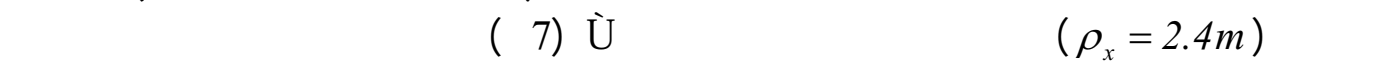

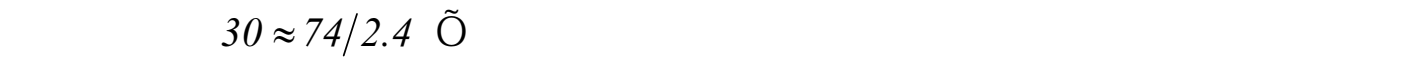

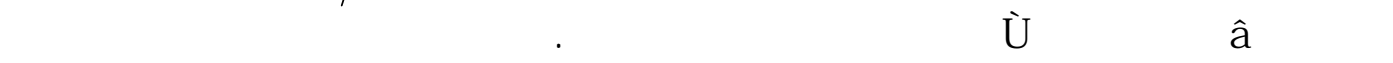

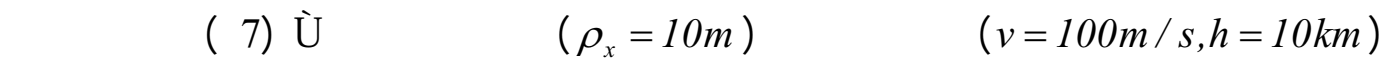

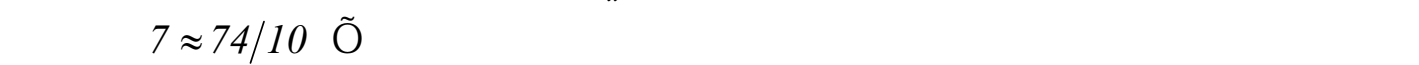

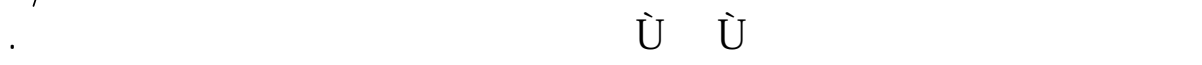

Conclusions:

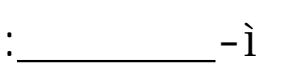

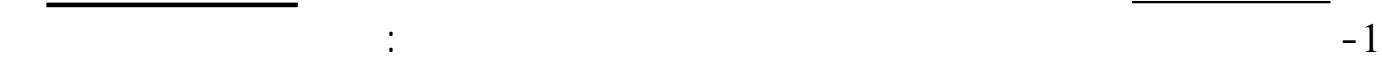

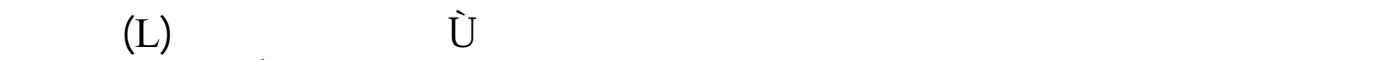

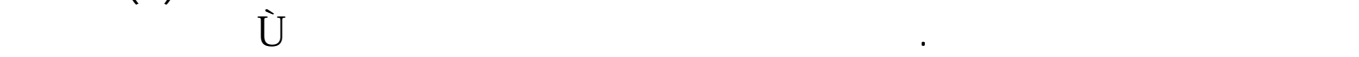

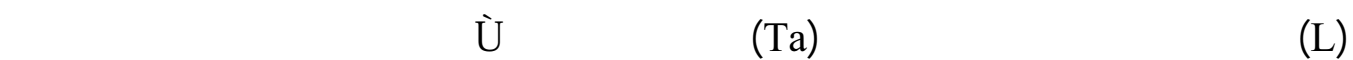

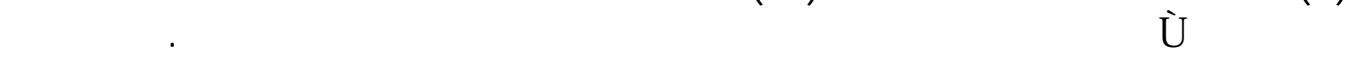

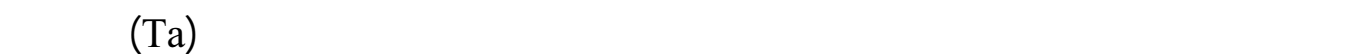
Gd

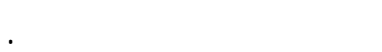

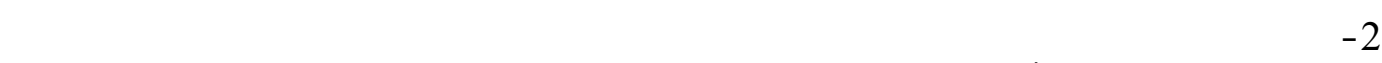

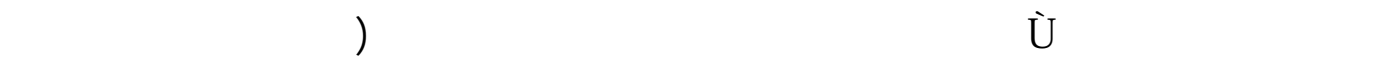

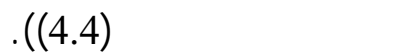




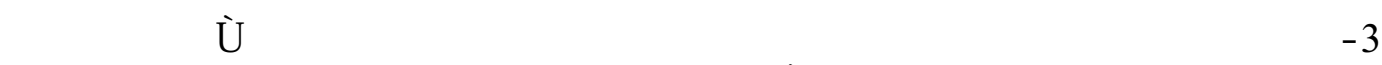

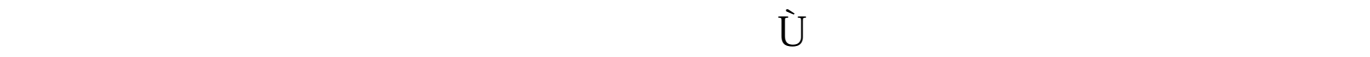

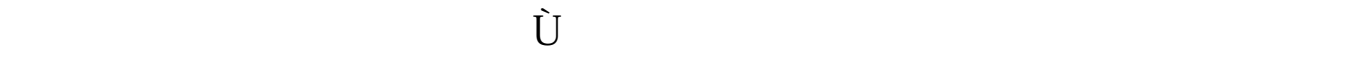

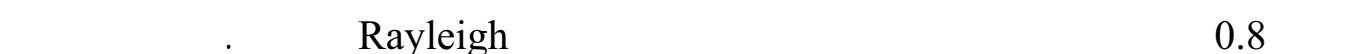
řás Ś

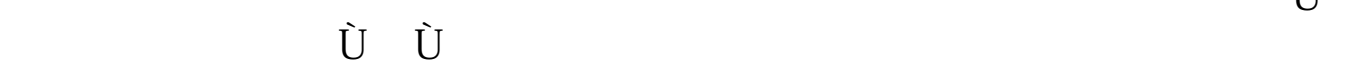

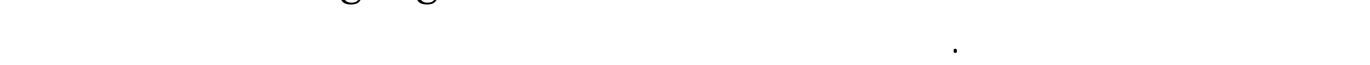

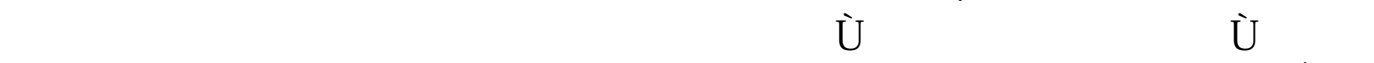

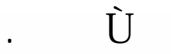

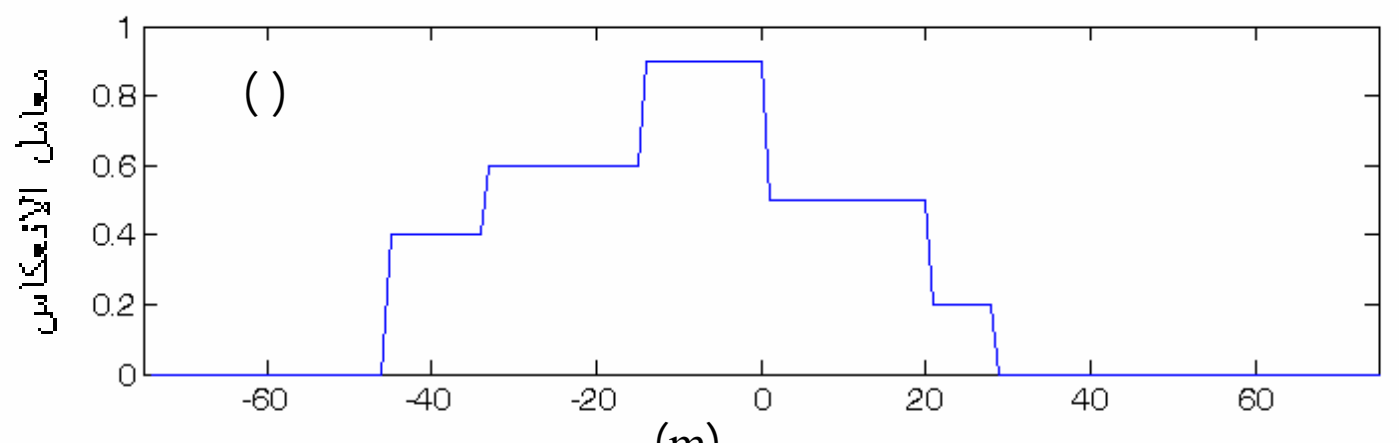

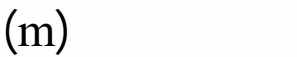
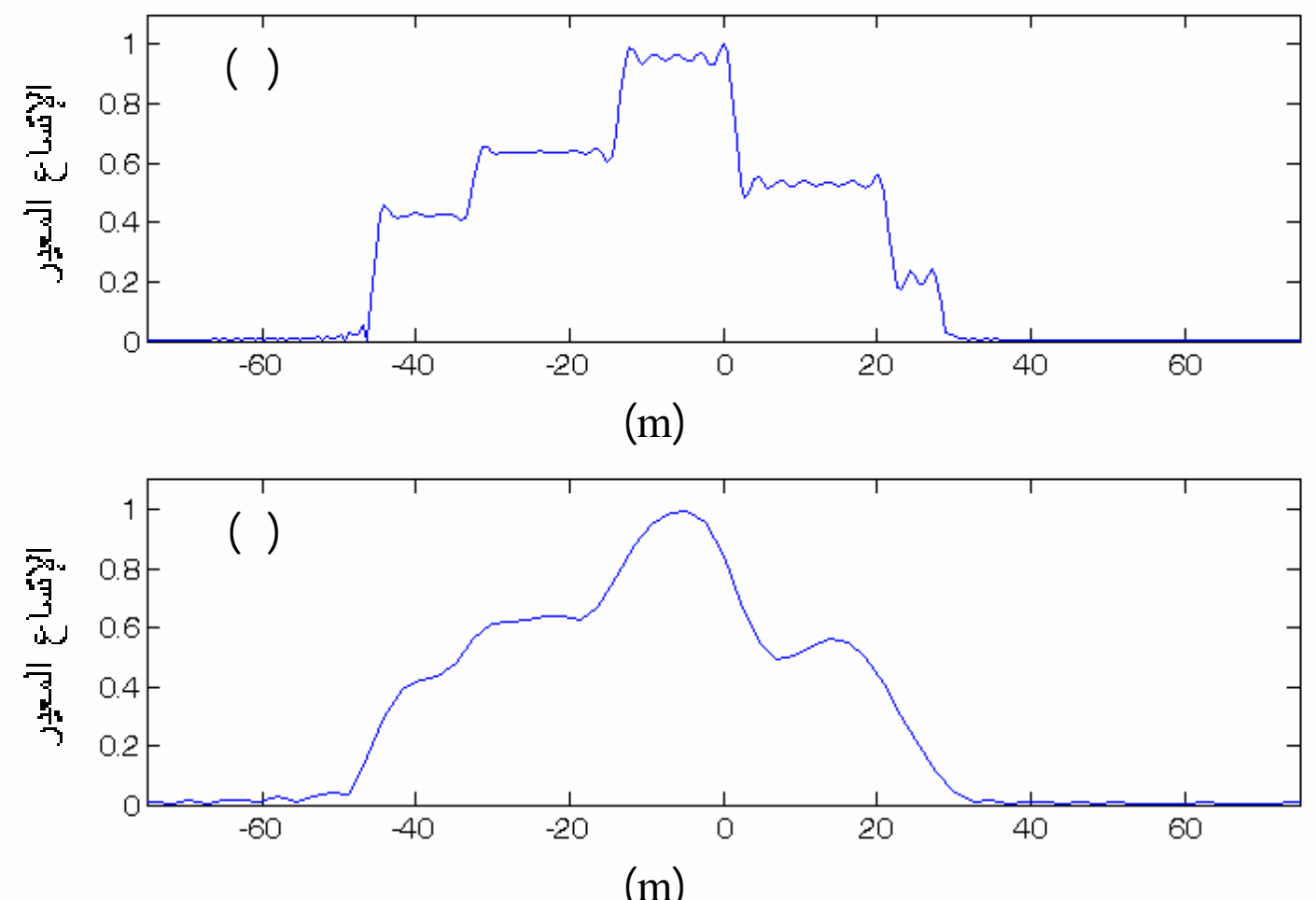

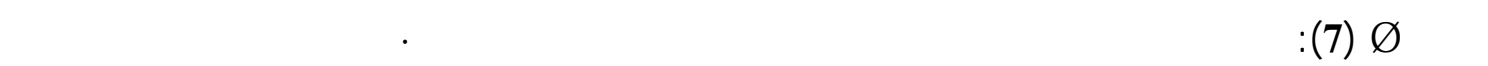

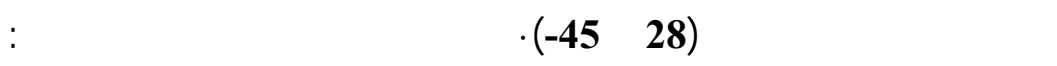

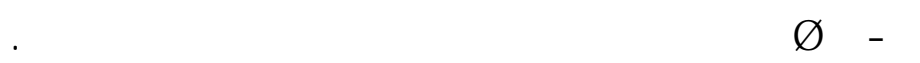

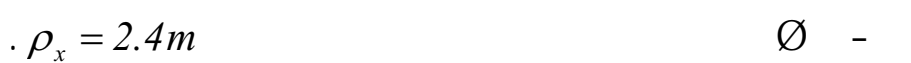

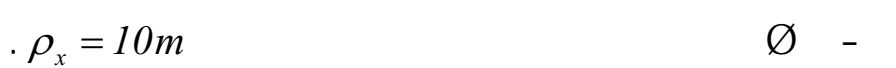




\section{$\underline{\text { References: }}$}

[1] Franceschetti, G., and Lanari, R., "Synthetic Aperture Radar Processing", CRC press, LLC, 1999.

[2] Lillesand, T. M., and Kiefer, R. W., "Remote Sensing and Image Interpretation“, New York, John Wiley\& Sons, Inc., Second Edition, 1987.

[3] "Radar Remote Sensing", http://www.rss.chalmers.se/rsg/News/Lundin/Chapter3.pdf

[4] Levanon, N., "Radar Principles", USA, John Wiley \&. Sons, Inc., 1988.

[5] Morris, G. V., "Airborne Pulsed Doppler Radar", Artech House, INC., 1988.

[6] Borden, B. and Cheney, M. ," Synthetic aperture radar imaging from high Doppler resolution measurements", Institute of Physics Publishing, Inverse Problems, 21, 2005, pp. 1-11.

[7] Richards, M. A., Lunsford, G.H., and Green Jr., A. H., "Modeling of Synthetic Aperture Radar for Nonconventional Geometry", IEEE International Radar Conference, 1985, p.366.

[8] Soumekh, M., "Reconnaissance with Ultra Wideband UHF Synthetic Aperture Radar", IEEE signal Processing Magazine, Vol. 12, No. 4, July 1995, pp 21-40.

[9] Borne, M. and Wolf, E., "Principles of Optics", Pergamon press, $7^{\text {th }}$ Ed., $1975, \mathrm{p} 430$.

[10] Chevalier, F. Le.," Principles of radar and sonar signal processing", Artech House, 2002.

[11]Christopher, R. ,"Imaging Radar",Chapter8， Radar， 9\17\2002, http://www.physics.nps.navy.mil/syllabus.pdf

[12] Umehara, T. et al., " Development of Airborne High-Resolution MultiParameter Imaging Radar SAR, Pi-SAR", Journal of the Communication research Lab.,Japan, Vol. 49, No. 2, 2002, pp. 109-126.

http://www2.crl.go.jp/kk/e414/shuppan/kihou-journal/journal-vol49no2/3$\underline{05 . p d f}$

[13] Thomson-CSF, "Side Looking Airborne Radar System", Catalogue, 1987.

[14] Tsunoda, S. I., et al., "Lynx: A High-Resolution Synthetic Aperture Radar", SPIE Aero Science, 1999, Vol.3704, http:// www.sandia.gov/RADAR/files/spie lynx.pdf.

[15] Brenner, A. and Ender, J.,"Very wideband radar imaging with air-borne SAR sensor PAMIR", Proc. IGRASS, Toulose, France, 21-25 July, 2000. 